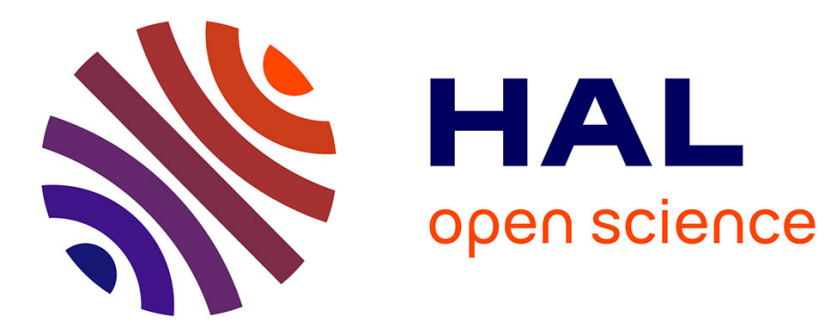

\title{
Analysis of control relevant nonlinear coupled nonlinear oscillatory systems
}

\author{
Ioan Doré Landau, Fethi Bouziani, Robert Bitmead, Alina Voda
}

\section{To cite this version:}

Ioan Doré Landau, Fethi Bouziani, Robert Bitmead, Alina Voda. Analysis of control relevant nonlinear coupled nonlinear oscillatory systems. European Journal of Control, 2008, 14 (4), pp.1-20. hal00384451

\section{HAL Id: hal-00384451 \\ https://hal.science/hal-00384451}

Submitted on 15 May 2009

HAL is a multi-disciplinary open access archive for the deposit and dissemination of scientific research documents, whether they are published or not. The documents may come from teaching and research institutions in France or abroad, or from public or private research centers.
L'archive ouverte pluridisciplinaire HAL, est destinée au dépôt et à la diffusion de documents scientifiques de niveau recherche, publiés ou non, émanant des établissements d'enseignement et de recherche français ou étrangers, des laboratoires publics ou privés. 


\title{
Analysis of control relevant coupled nonlinear oscillatory systems
}

\author{
Ioan D. Landau ${ }^{A *} \quad$ Fethi Bouziani $^{A}$ \\ Robert R. Bitmead ${ }^{B} \quad$ Alina Voda-Besançon ${ }^{A}$ \\ ${ }^{A}$ Département d'Automatique, GIPSA-Lab, ENSIEG BP 46, 38402 Saint-Martin d'Hères, France \\ ${ }^{B}$ Mechanical \& Aerospace Engineering Department, University of California, San Diego \\ La Jolla CA 92093-0411, USA
}

\begin{abstract}
The paper proposes and analyzes two prototype structures of coupled generalized van der Pol equations able to describe self-excitation of simultaneous oscillations with distinct frequencies. These structures are relevant for describing oscillations phenomena which may be encountered on systems subject to control. These structures are analyzed using the Krylov-Bogoliubov averaging method. This analysis allows to establish conditions for the occurrence of the various operation regimes. The usefulness of the results is illustrated by their application to the straightforward analysis of the properties of a combustion instability model.
\end{abstract}

\section{keywords}

nonlinear oscillating systems, van der Pol equation, coupled oscillators, Krylov-Bogoliubov method.

\section{Introduction}

Presence of oscillatory phenomena in control systems is well acknowledged. One can distinguish two basic situations:

- Oscillatory phenomena are inherent to the plant to be controlled.

- Oscillatory phenomena occurs in the feedback control system.

These phenomena are generated by feedback (existing inside the plant or through the feedback control) and they are described by nonlinear equations ${ }^{1}$. It is important to note that in many systems to be controlled these phenomena are often considered as "disturbances" to be attenuated by the control system and the generating model of these oscillations is ignored.

Such an example is in mechanical systems where in many situations these oscillations have the generic term "vibrations" and for which a large variety of control strategies have been developed in order to reduce them [2]. Similarly

* Corresponding author, Phone:0033476826391, Fax:0033476826388, E-mail:landau@lag.ensieg.inpg.fr.

${ }^{1}$ linear models neglect important nonlinear phenomena as saturation phenomenon which explain steady oscillation with fixed amplitude 
in combustion systems where thermo-acoustic oscillations may occurs (generic term: combustion instability), a number of control strategies have been developed by considering these oscillations as disturbances to be attenuated [1] (and ignoring the model behind the generation of these oscillations). However in many cases a total different approach can be considered:

- one can model the oscillatory phenomena,

- one can investigate strategies for quenching the oscillatory phenomena taking advantage of the nonlinear feature of the model describing the phenomena.

Nevertheless, the first stage in this approach is to establish a relevant model (which of course is nonlinear) and analyze its various operation regimes (i.e understanding when and how oscillations occurs).

From the point of view of modelling of such phenomena one have to combine two approaches:

1. The model should reflect the phenomena encountered in practice.

2. The basic mathematical models for describing oscillatory phenomena are van der Pol (Rayleigh, Duffing) type equations.

As a consequence these models will be often built by using modified and coupled van der Pol (Rayleigh, Duffing) equations (in order to capture simultaneous oscillatory phenomena with distinct frequencies) $[6,8,10,15,16,18]$. For building such modified and coupled equations one has to remark that van der Pol equations correspond to a nonlinear feedback path around a pure linear resonator. The modifications will be done essentially in the feedback path which may become very complex by combining static nonlinearities with linear dynamics. These models can be subject to external excitations or control inputs which enter linearly or multiplicatively.

The resulting nonlinear equations describing such oscillatory systems do not have in general an exact solution and therefore the problem of the analytical analysis of their behavior is fundamental.

Numerical investigation while used sometimes [14] is not a satisfactory approach because on one hand the phenomena should be understood and on the other hand it is difficult to cover all range of possible variations of the parameters (one may skip some critical combination of parameters values). What is needed, is an analytical study of the behavior of these oscillatory system which is also fundamental for developing in a later stage a "quenching" policy.

The theory of oscillatory systems is a well established field. See for example [3, 9, 11, 12]. A number of approximation methods have been proposed for studying the properties of the equations describing oscillatory systems. However among all these methods, it seems that the averaging method of Krylov and Bogoliubov (in short K-B method) is one of the most efficient and with probably the most widely application range [3, 12].

The objective of this paper is to analyze two classes of coupled oscillatory systems relevant for describing systems which can feature self-excitation of single or multiple simultaneous oscillations of distinct frequencies and may be subject to an external excitation. This investigation will be done using the K-B method which will be briefly reviewed from the perspective of problems encountered in control.

The major original contributions of this paper are: 
1. Introduction of two prototype structures of coupled generalized van der Pol equations.

2. Analysis of the behavior of these structures using the K-B method.

3. Application of the results to the straightforward analysis of a model used for describing combustion instabilities.

This paper will not discuss the problem of "quenching" of oscillations through a control strategies. This will be the object of a distinct paper. Preliminary results can be found in [4].

The paper is organized as follows: In Section 2 the K-B approximation method for autonomous multi-resonator systems is briefly reviewed and its use is illustrated in Section 3 for the analysis of the generalized van der Pol equation. In section 4 a first structure of coupled generalized van der Pol equations is analyzed using the K-B method. The various regimes of operation are emphasized and conditions for their occurrence are provided. The case when this system is driven by an external periodic excitation is examined in Section 5. In Section 6 a further generalization of the coupling between two resonators is considered. This structure can feature simultaneous non-harmonic oscillations. Finally in Section 6 it is shown that a model for combustion instabilities can be brought to the prototype structure considered in this section and therefore the existence of simultaneous non harmonic oscillations observed in practice receives a theoretical explanation. Some concluding remarks are presented in Section 7.

\section{K-B approximation for autonomous multi-resonators systems}

Consider a system with $n$ resonators described by differential equations of the form,

$$
\frac{d^{2} x_{j}}{d t^{2}}+\omega_{j}^{2} x_{j}=\varepsilon f_{j}\left(x, \frac{d x}{d t}\right),(j=1,2, \ldots, n),
$$

where $x=\left\{x_{1}, \ldots, x_{n}\right\}, \frac{d x}{d t}=\left\{\frac{d x_{1}}{d t}, \ldots, \frac{d x_{n}}{d t}\right\}$ and $\varepsilon$ is a small parameter. The systems governed by (1) belong to near conservative system class, where function $f_{j}$ is called perturbation term of equation $j$. For the $j$ th resonator, the first K-B approximation (for more details see Chapter 2 of [12]) proposes the solution

$$
x_{j}=a_{j} \cos \left(\psi_{j}\right),
$$

where $\psi_{j}=\omega_{j} t+\theta_{j}, a_{j}$ and $\theta_{j}$ are slowly time-varying functions obeying the equations

$$
\left\{\begin{array}{l}
\frac{d a_{j}}{d t}=-\frac{\varepsilon}{2 \omega_{j}} H_{j j}\left(a_{1}, \ldots, a_{n}, \theta_{1}, \ldots, \theta_{n}\right), \\
\frac{d \theta_{j}}{d t}=-\frac{\varepsilon}{2 \omega_{j} a_{j}} G_{j j}\left(a_{1}, \ldots, a_{n}, \theta_{1}, \ldots, \theta_{n}\right),
\end{array}\right.
$$

with $H_{j j}$ and $G_{j j}$ obtained from the function $f_{j}\left(x, \frac{d x}{d t}\right)$ by substituting

$$
\left\{\begin{array}{l}
x_{k}=a_{k} \cos \left(\omega_{k} t+\theta_{k}\right), \\
\frac{d x_{k}}{d t}=-a_{k} \omega_{k} \sin \left(\omega_{k} t+\theta_{k}\right),
\end{array}(k=1,2, \ldots, n)\right.
$$

and by setting it in the form

$$
\begin{aligned}
& f_{j}\left(a_{1} \cos \left(\omega_{1} t+\theta_{1}\right), \ldots, a_{n} \cos \left(\omega_{n} t+\theta_{n}\right),-a_{1} \omega_{1} \sin \left(\omega_{1} t+\theta_{1}\right), \ldots,-a_{n} \omega_{n} \sin \left(\omega_{n} t+\theta_{n}\right)\right) \\
& =H_{j j} \sin \left(\omega_{j} t+\theta_{j}\right)+G_{j j} \cos \left(\omega_{j} t+\theta_{j}\right)+\sum_{\omega_{j} \nsucc \omega_{\ell}}^{r}\left(H_{\ell j} \sin \left(\omega_{\ell} t+\theta_{\ell}\right)+G_{\ell j} \cos \left(\omega_{\ell} t+\theta_{\ell}\right)\right),
\end{aligned}
$$




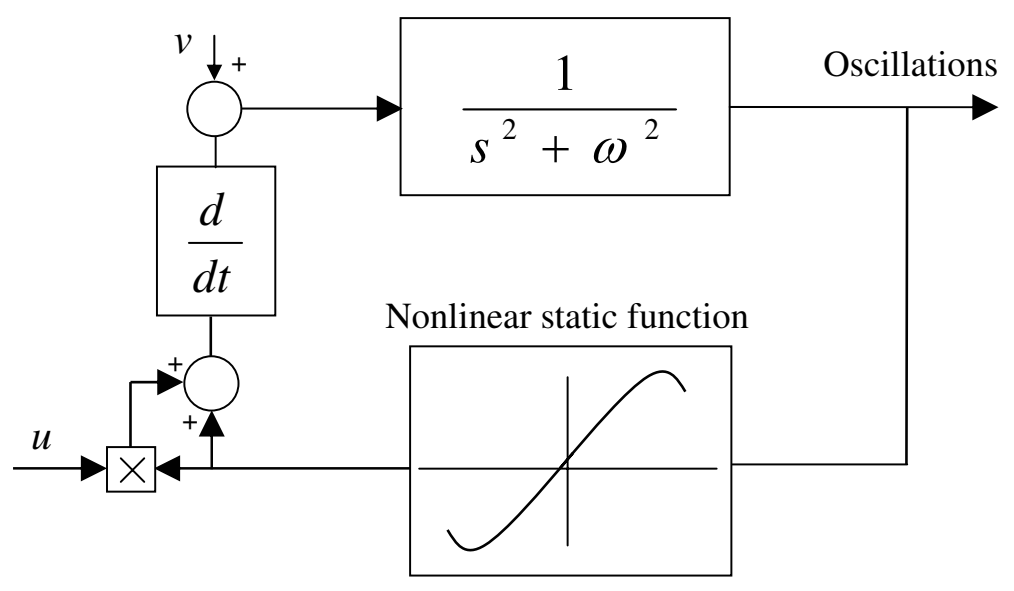

Figure 1: Generalized van der Pol equation in feedback structure with multiplicative control

where $\omega_{\ell}$ and $\theta_{\ell}$ are integer linear combinations of $\omega_{1}, \ldots, \omega_{n}$ and $\theta_{1}, \ldots, \theta_{n}$, respectively, and $r$ is the number of possible integer linear combinations of $\omega_{1}, \ldots, \omega_{n}$ different from $\omega_{j}$. For $x_{j}$ the coefficients of the fundamental term in (5) are used and the all other terms are eliminated.

\section{Generalized van der Pol equation}

In nonlinear oscillating systems control field, the determination of plant model is a fist step of control tuning. Establishing a relevant model for nonlinear oscillating systems requires good knowledge of the physical phenomenon at the same time as the availability of mathematical tools allowing the analysis of the model. The Krylov-Bogoliubov (K-B) method (detailed in $[3,9,11,12,13]$ ) is such an analytical method allowing an efficient analysis of nonlinear oscillating systems. The effectiveness of this method combined with relevant models may be considered as a major step forward in modelling self-oscillating systems.

The multiplicative control leads in general to some difficulties in analysis. However in some situations, this control presents important advantages since the multiplicative input often acts directly on the system part which cause instability. For unstable nonlinear resonators cases, the instability can be caused by negative stiffness or damping. The negative stiffness situation is encountered in inverted pendulum problem driven by vertical excitation [17], and the negative damping situation is encountered combustion instabilities models [7, 4].

The van der Pol equation is one of most known nonlinear self-oscillatory system characterized by a negative damping. The basic van der Pol equation is the following:

$$
\ddot{x}+\omega^{2} x=\varepsilon \frac{d}{d t}\left\{x-\frac{1}{3} x^{3}\right\},
$$

where $\varepsilon$ is a small parameter and $\omega$ is the frequency of oscillation. If we generalize the form of the right hand of (6), one obtains

$$
\ddot{x}+\omega^{2} x=\frac{d}{d t}\left\{\varphi_{0}+\varphi_{1} x-\frac{\varphi_{3}}{3} x^{3}\right\},
$$


where $\varphi_{1}$ and $\varphi_{3}$ are arbitrary positive constants and $\varphi_{0}$ is arbitrary constant.

The self-oscillatory nature is due to the right hand of (7) corresponding to a nonlinear damping with negative values about the origin. Therefore, for control one proposes to introduce the multiplicative action $u$ on the nonlinear damping which causes the system instability. Moreover, an additive input $v$ can be introduced. This input can be considered as noise excitation.

$$
\ddot{x}+\omega^{2} x=\frac{d}{d t}\left\{(1+u)\left(\varphi_{0}+\varphi_{1} x-\frac{\varphi_{3}}{3} x^{3}\right)\right\}+v,
$$

The generalized van der Pol equation, can be seen as a system having a closed loop structure. The feedforward path is characterized by one linear resonator in feedback with a nonlinear path which creates a negative damping leading to the excitation of oscillations. The nonlinear path represents the differentiation of a nonlinear static function with a drift equal to $\varphi_{0}$, a slope about the origin equal to $\varphi_{1}$ and a level of saturation phenomenon illustrated by $\varphi_{3}$. Figure 1 presents the closed loop system structure with multiplicative control associated to equation (8).

We will proceed next to the analysis of equation (7) using the K-B method. The analysis is not new, but it will illustrate how the methodology will be used for more complex systems which will be considered in the subsequent sections.

\subsection{K-B analysis}

The application of K-B approximation need to consider that structure (7) belong to near conservative system class (form (1) in mono-resonator case), where the perturbation term is the following function (with $\varepsilon=1$ )

$$
f=\frac{d}{d t}\left\{\varphi_{0}+\varphi_{1} x-\frac{\varphi_{3}}{3} x^{3}\right\},
$$

Introducing a structure for the solution of the form

$$
\left\{\begin{array}{l}
x=a \cos (\omega t+\theta), \\
\frac{d x}{d t}=-a \omega \sin (\omega t+\theta),
\end{array}\right.
$$

into (9), one gets

$$
f=-\omega \varphi_{1} a\left(1-\varphi_{3}(a \cos (\omega t+\theta))^{2}\right) \sin (\omega t+\theta),
$$

To approximate the solution of (7), it is necessary to set (11) in the form (5) (in mono-resonator case). The application of trigonometric simplifications on (11) yields

$$
f=-\varphi_{1} \omega a\left(1-\frac{\varphi_{3}}{4 \varphi_{1}} a^{2}\right) \sin (\omega t+\theta)-\frac{\varphi_{3}}{4} a^{3} \sin (3 \omega t+3 \theta),
$$

From this expression one can deduces easily

$$
\left\{\begin{array}{l}
H_{1}=-\varphi_{1} \omega a\left(1-\frac{\varphi_{3}}{4 \varphi_{1}} a^{2}\right), \\
G_{1}=0
\end{array}\right.
$$




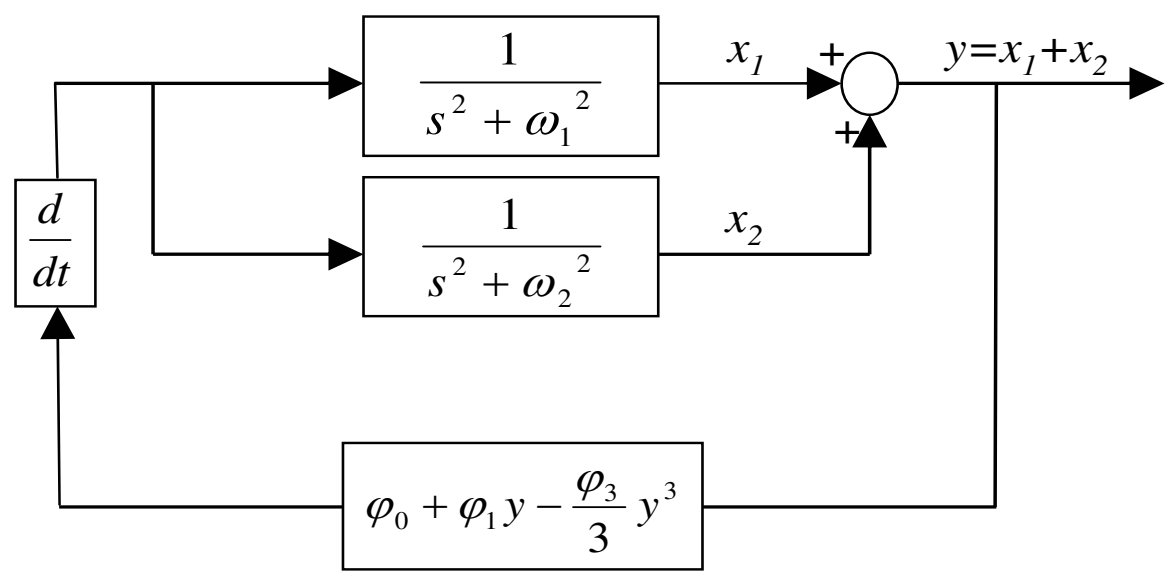

Figure 2: Two coupled generalized van der Pol equations

By applying the rules (3) on (13), one obtains finally

$$
\left\{\begin{array}{l}
\frac{d a}{d t}=\frac{\varphi_{1} a}{2}\left(1-\frac{\varphi_{3}}{4 \varphi_{1}} a^{2}\right), \\
\frac{d \theta}{d t}=0
\end{array}\right.
$$

The equations (14) possesses two steady-state solutions. The first at the origin is unstable (since $\varphi_{1}>0$ ). The second steady-state solution which corresponds to amplitude $a_{s}=2 \sqrt{\frac{\varphi_{1}}{\varphi_{3}}}$ is stable. This implies a periodic oscillation with frequency equal to $\omega$ and amplitude which will converge necessarily to $2 \sqrt{\frac{\varphi_{1}}{\varphi_{3}}}$. The result is predictable by examination of the right hand of (14). Indeed, one sees that for initial value $a<a_{s}$, the damping is negative so oscillation will grow. But once $a=a_{s}$, the damping is equal to zero so this will correspond to oscillations of constant amplitude. On the other hand, for the initial value $a>a_{s}$, the damping is positive and the oscillations will decrease till the right hand of (14) will become zero for $a=a_{s}$.

\section{Two coupled generalized van der Pol equations}

In this section the interaction of two self-oscillatory systems will be analyzed. By such analysis, we will answer two important questions:

- What happen if two self-oscillatory systems such as van der Pol equations are associated with same coupled term?

- In what conditions, the oscillations of both equations coexist? 
We consider the two coupled generalized van der Pol equations structure presented in Figure 2 and described by the following differential equations

$$
\left\{\begin{array}{c}
\frac{d^{2} x_{1}}{d t^{2}}+\omega_{1}^{2} x_{1}=\frac{d}{d t}\left(\varphi_{0}+\varphi_{1} y-\frac{\varphi_{3}}{3} y^{3}\right) \\
\frac{d^{2} x_{2}}{d t^{2}}+\omega_{2}^{2} x_{2}=\frac{d}{d t}\left(\varphi_{0}+\varphi_{1} y-\frac{\varphi_{3}}{3} y^{3}\right) \\
y=x_{1}+x_{2}
\end{array}\right.
$$

where $\omega_{1}$ and $\omega_{2}$ are the natural radian frequencies of the first and second resonators respectively and which can have arbitrary values with some modest provisions to be developed.

\subsection{K-B analysis}

Consider the equations system (15) and the form (1) (with $\varepsilon=1$ ), in this case

$$
\begin{aligned}
f_{1}=f_{2}=f= & \frac{d}{d t}\left(\varphi_{0}+\varphi_{1}\left(x_{1}+x_{2}\right)-\frac{\varphi_{3}}{3}\left(x_{1}+x_{2}\right)^{3}\right) \\
& =\varphi_{1}\left(1-\frac{\varphi_{3}}{\varphi_{1}}\left(x_{1}+x_{2}\right)^{2}\right)\left(\frac{d x_{1}}{d t}+\frac{d x_{2}}{d t}\right) .
\end{aligned}
$$

Introducing

$$
\left\{\begin{array}{l}
x_{i}=a_{i} \cos \left(\omega_{i} t+\theta_{i}\right), \\
\frac{d x_{i}}{d t}=-a_{i} \omega_{i} \sin \left(\omega_{i} t+\theta_{i}\right),
\end{array} \quad(i=1,2)\right.
$$

into (16) and using trigonometric simplifications, one gets

$$
\begin{aligned}
& f=\varphi_{1}\left\{-\omega_{1} a_{1}\left(1-\frac{\varphi_{3}}{\varphi_{1}}\left(\frac{a_{1}^{2}}{4}+\frac{a_{2}^{2}}{2}\right)\right) \sin \left(\omega_{1} t+\theta_{1}\right)-\omega_{2} a_{2}\left(1-\frac{\varphi_{3}}{\varphi_{1}}\left(\frac{a_{2}^{2}}{4}+\frac{a_{1}^{2}}{2}\right)\right) \sin \left(\omega_{2} t+\theta_{2}\right)\right. \\
& +\frac{\varphi_{3}}{\varphi_{1}}\left[\omega_{1} \frac{a_{1}^{3}}{4} \sin \left(3\left(\omega_{1} t+\theta_{1}\right)\right)+\omega_{2} \frac{a_{2}^{3}}{4} \sin \left(3\left(\omega_{2} t+\theta_{2}\right)\right)+\left(2 \omega_{1}+\omega_{2}\right) \frac{a_{1}^{2} a_{2}}{4} \sin \left(\left(2 \omega_{1}+\omega_{2}\right) t+2 \theta_{1}+\theta_{2}\right)\right. \\
& +\left(\omega_{1}+2 \omega_{2}\right) \frac{a_{1} a_{2}^{2}}{4} \sin \left(\left(\omega_{1}+2 \omega_{2}\right) t+\theta_{1}+2 \theta_{2}\right)+\left(2 \omega_{1}-\omega_{2}\right) \frac{a_{1}^{2} a_{2}}{4} \sin \left(\left(2 \omega_{1}-\omega_{2}\right) t+2 \theta_{1}-\theta_{2}\right) \\
& \left.\left.+\left(2 \omega_{2}-\omega_{1}\right) \frac{a_{2}^{2} a_{1}}{4} \sin \left(\left(2 \omega_{2}-\omega_{1}\right) t+2 \theta_{2}-\theta_{1}\right)\right]\right\} .
\end{aligned}
$$

from which one can see the existence of the frequency set

$$
W=\left\{\omega_{1}, \omega_{2}, 3 \omega_{1}, 3 \omega_{2}, 2 \omega_{1}+\omega_{2}, \omega_{1}+2 \omega_{2}, 2 \omega_{1}-\omega_{2}, 2 \omega_{2}-\omega_{1}\right\} .
$$

This set is very important for finding the possible operation regimes of the system, i.e. for $x_{1}$ (respectively $x_{2}$ ), the remaining terms from (18) after application of the K-B approximation will only be the terms with the frequency $\omega$ from $W$ such as $\omega \approx \omega_{1}$ (respectively $\omega_{2}$ ). Consequently, one has the following classification, which will be elaborated and explained shortly :

1. $\omega_{1} \not \approx\left\{\omega_{2}, 3 \omega_{2}, \frac{\omega_{2}}{3}\right\}$-two generators with competitive quenching

2. $\omega_{1} \approx \omega_{2}$-mutual synchronization with close frequencies

3. $\omega_{1} \approx 3 \omega_{2}$ (respectively $\omega_{2} \approx 3 \omega_{1}$ )-mutual synchronization with multiple harmonics 


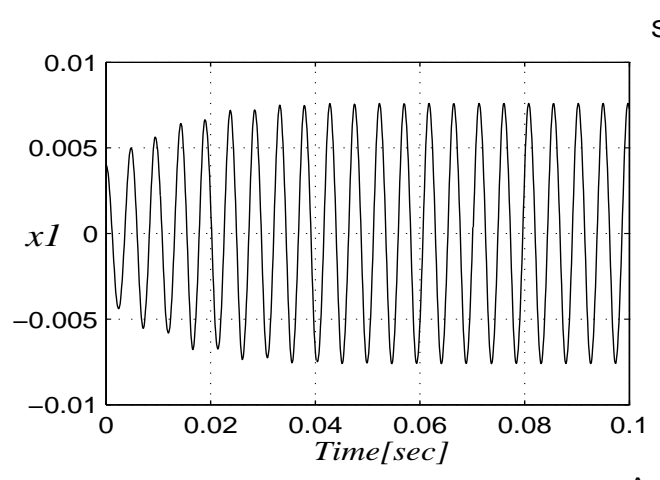

Simulation

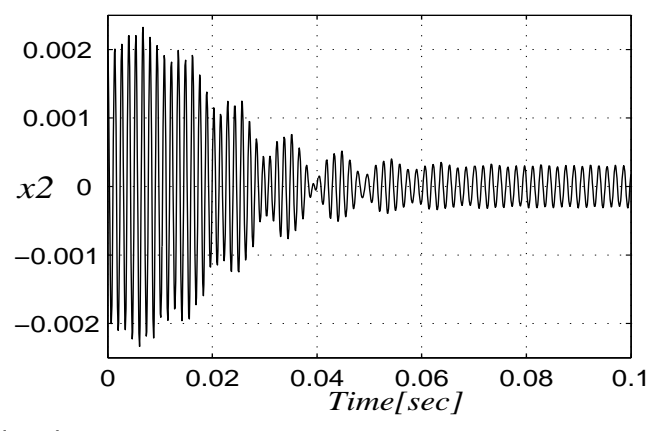

Approximation
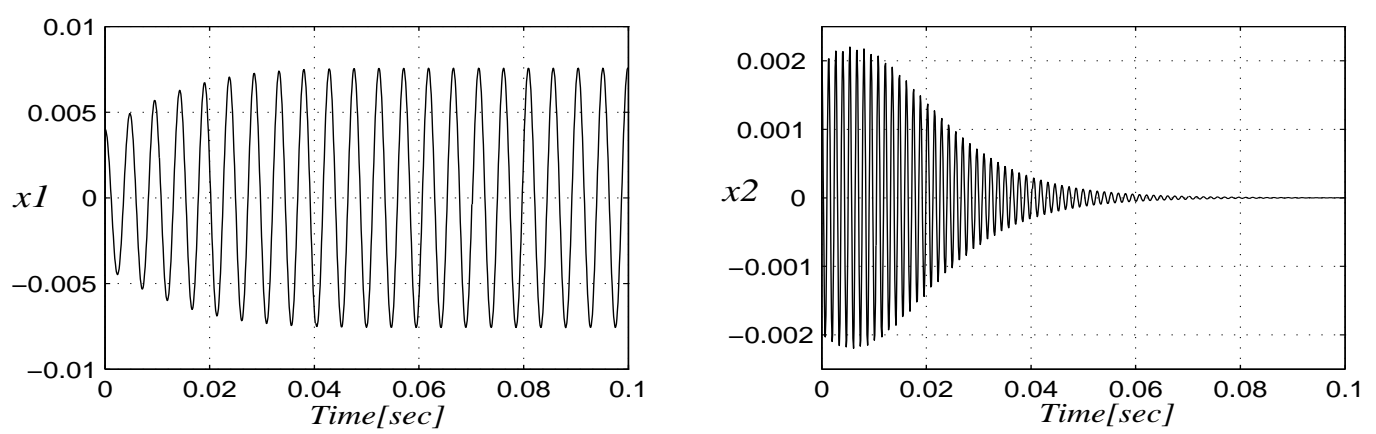

Figure 3: Simulation test of the system (15) and the approximations (21) and (22) with $\omega_{1}=$ $2 \pi \times 210, \omega_{2}=2 \pi \times 740, \varphi_{1}=1.78 \times 10^{2}, \varphi_{3}=1.24 \times 10^{7}, a_{1}(0)=4 \times 10^{-3}$ and $a_{2}(0)=2 \times 10^{-3}$.

\subsubsection{Two generators with competitive quenching}

In the case where the frequencies $\omega_{1}$ and $\omega_{2}$ respect Condition 1 above, there is no interconnection effect between the both frequencies and the K-B approximation uses only the fundamental oscillations terms of $f(.,$.$) . Furthermore,$ $\mathrm{K}-\mathrm{B}$ approximation gives the result presented in the following lemma.

\section{Lemma 1}

Consider the case where the frequencies $\omega_{1}$ and $\omega_{2}$ satisfy the condition

$$
\omega_{1} \not \approx\left\{\omega_{2}, 3 \omega_{2}, \frac{\omega_{2}}{3}\right\} .
$$

Consequently the approximated solutions of (15) are

$$
\begin{aligned}
& x_{i}=a_{i} \cos \left(\omega_{i} t+\theta_{i}\right), \quad(i=1,2) \\
& \text { with } \\
& \left\{\begin{array}{l}
\frac{d a_{1}}{d t}=\frac{\varphi_{1}}{2} a_{1}-\frac{\varphi_{3}}{2} a_{1}\left(\frac{a_{1}^{2}}{4}+\frac{a_{2}^{2}}{2}\right), \\
\frac{d a_{2}}{d t}=\frac{\varphi_{1}}{2} a_{2}-\frac{\varphi_{3}}{2} a_{2}\left(\frac{a_{2}^{2}}{4}+\frac{a_{1}^{2}}{2}\right), \\
\frac{d \theta_{1}}{d t}=0 \\
\frac{d \theta_{2}}{d t}=0
\end{array}\right.
\end{aligned}
$$


The steps leading to this lemma are presented in Appendix A. Let us find steady-state solutions of (22). In this case, (22) possesses four steady-state solutions

$$
\begin{gathered}
a_{1 s}=0 \text { and } a_{2 s}=0, \\
a_{1 s}=\frac{2}{\sqrt{3}} \sqrt{\frac{\varphi_{1}}{\varphi_{3}}} \text { and } a_{2 s}=\frac{2}{\sqrt{3}} \sqrt{\frac{\varphi_{1}}{\varphi_{3}}}, \\
a_{1 s}=2 \sqrt{\frac{\varphi_{1}}{\varphi_{3}}} \text { and } a_{2 s}=0, \\
a_{1 s}=0 \text { and } a_{2 s}=2 \sqrt{\frac{\varphi_{1}}{\varphi_{3}}} .
\end{gathered}
$$

Both former solutions (23) and (24) are unstable, and both latter solutions (25) and (26) are stable. Therefore, the amplitudes of $x_{1}$ and $x_{2}$ converge to one of both possible stationary states (25) and (26). Depending on the initial condition, one of the generators is excited, while the oscillations of the other generator are entirely quenched. Such quenching of the oscillations of one of the generators, caused by the sufficiently large non-linear coupling between them, is known as competitive quenching.

It was noted that, if $a_{1}(0)>a_{2}(0), x_{1}$ is excited and the oscillations of $x_{2}$ are entirely quenched, and the converse effect occurs when $a_{1}(0)<a_{2}(0)$. Figure 3 presents a simulation test of competitive quenching phenomenon, the upper part shows the outputs of (15) and lower part shows the outputs approximated by (21) and (22).

\subsubsection{Mutual synchronization with close frequencies}

In the case where the frequencies $\omega_{1}$ and $\omega_{2}$ are close, an important interaction exist between resonators. For $x_{1}$ (respectively $x_{2}$ ), one has the properties

$$
\begin{aligned}
& \omega_{1} \approx\left\{\omega_{2},\left|2 \omega_{1}-\omega_{2}\right|,\left|2 \omega_{2}-\omega_{1}\right|\right\}, \\
& \omega_{1} \not \approx\left\{3 \omega_{1}, 3 \omega_{2}, 2 \omega_{1}+\omega_{2}, \omega_{1}+2 \omega_{2}\right\},
\end{aligned}
$$

The application of K-B approximation implies the conservation of all coefficients of sinusoidal terms in $f_{i}$ with a frequency close to $\omega_{1}$ (respectively $\omega_{2}$ ) and the elimination of all other terms. Therefore, only the sinusoidal terms with frequencies $\omega_{1}, \omega_{2}, 2 \omega_{1}-\omega_{2}$ and $2 \omega_{2}-\omega_{1}$ will be employed in K-B approximation.

\section{Lemma 2}

Consider the case where the frequencies satisfy $\omega_{1} \approx \omega_{2}$. Consequently the approximated solutions of (15) are

$$
x_{i}=a_{i} \cos \left(\omega_{i} t+\theta_{i}\right), \quad(i=1,2)
$$

with $a_{1}, a_{2}, \theta_{1}$ and $\theta_{2}$ governed by

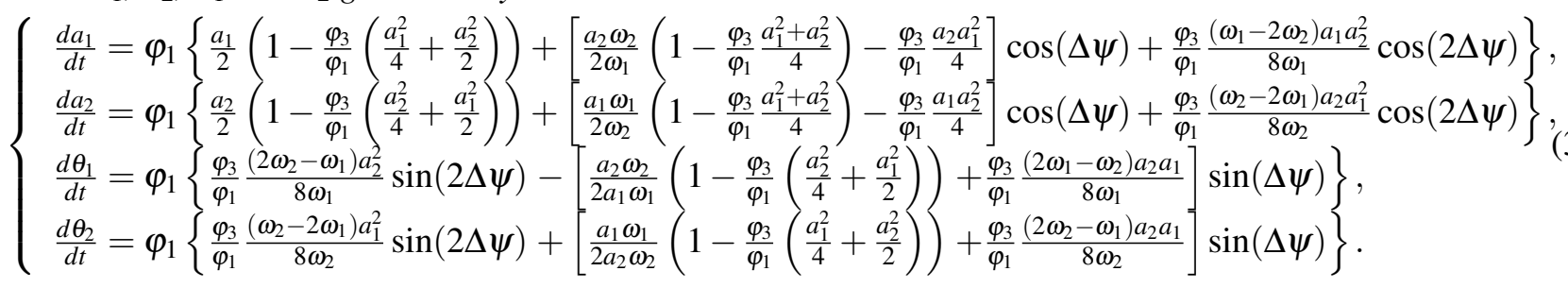

where

$\Delta \psi=\psi_{1}-\psi_{2}=\left(\omega_{1}-\omega_{2}\right) t+\theta_{1}-\theta_{2}$. 

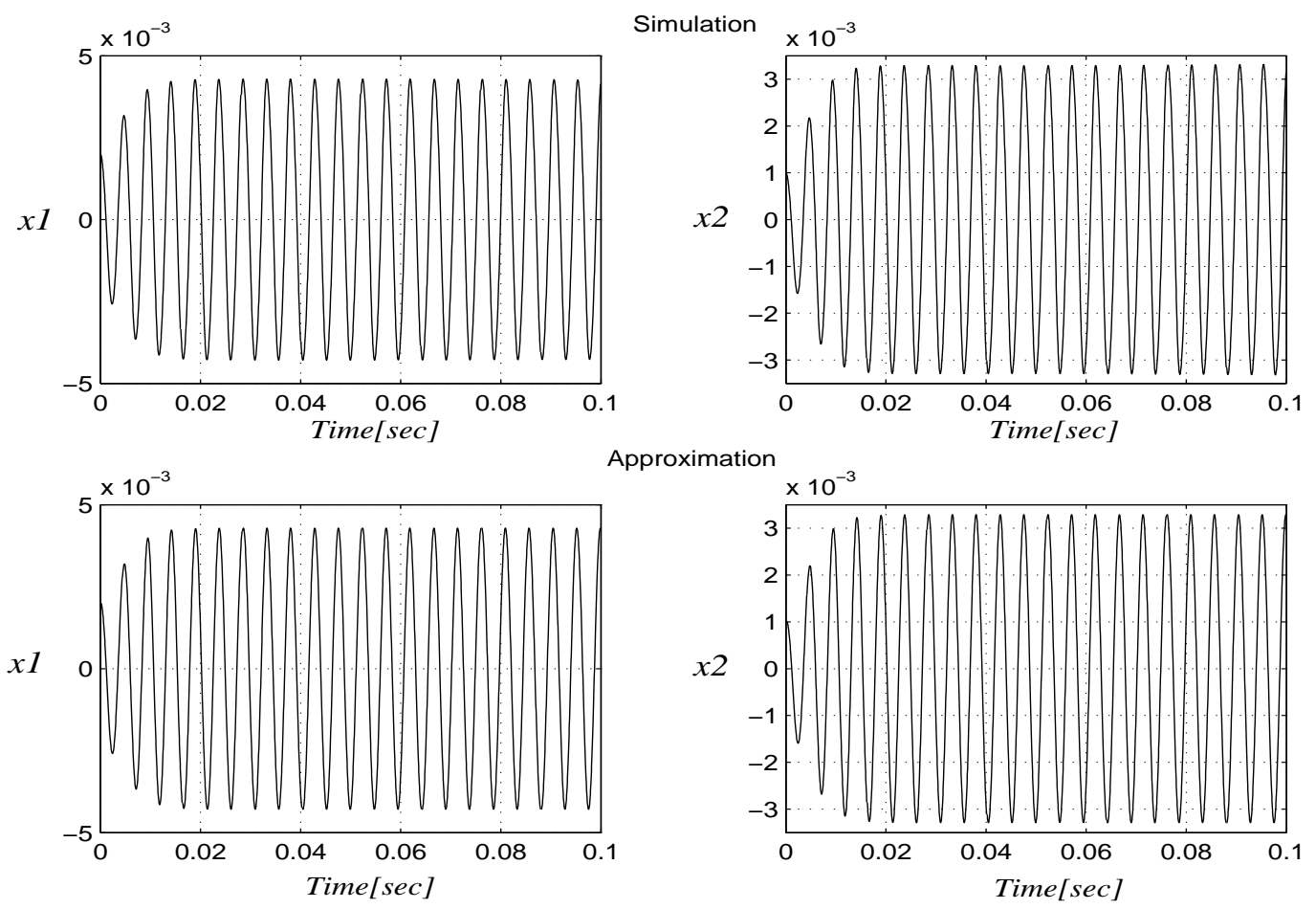

Approximation

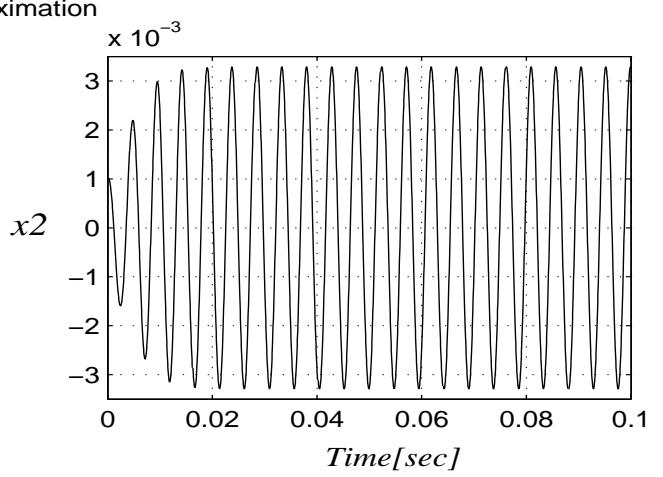

Figure 4: Simulation test of the system (15) and the approximations (29) and (30) with $\omega_{1}=$ $\omega_{2}=2 \pi \times 210, \varphi_{1}=1.78 \times 10^{2}, \varphi_{3}=1.24 \times 10^{7}, a_{1}(0)=2 \times 10^{-3}, a_{2}(0)=10^{-3}$ and $\theta_{1}(0)=\theta_{1}(0)=0$.

The proof of this lemma is presented in Appendix B. This result is very important, because in parallel with differential equation (15), it is possible to compute from (30) the amplitude and the phase evolutions of the output and to compare both to signals measured in practice.

The integration and study of stationary solutions of (30) are very difficult. However, to find the stationary solutions when $\omega_{1}=\omega_{2}=\omega$, one can adopt the following steps. Using $y=x_{1}+x_{2}$, if we add the both equations of (15) we obtain

$$
\begin{gathered}
\frac{d^{2}\left(x_{1}+x_{2}\right)}{d t^{2}}+\omega^{2}\left(x_{1}+x_{2}\right)=\frac{d}{d t}\left(\varphi_{0}+\varphi_{1}\left(x_{1}+x_{2}\right)-\frac{\varphi_{3}}{3}\left(x_{1}+x_{2}\right)^{3}\right) \\
\Rightarrow \frac{d^{2} y}{d t^{2}}+\omega^{2} y=\frac{d}{d t}\left(\varphi_{0}+\varphi_{1} y-\frac{\varphi_{3}}{3} y^{3}\right) .
\end{gathered}
$$

It is seen that (32) corresponds to generalized van der Pol equation (7) approximated in section 3 as follows

$$
y=2 \sqrt{\frac{\varphi_{1}}{\varphi_{3}}} \cos (\omega t+\theta) \Rightarrow x_{1}+x_{2}=2 \sqrt{\frac{\varphi_{1}}{\varphi_{3}}} \cos (\omega t+\theta),
$$

where $\theta$ is the arbitrary instantaneous phase, which satisfies

$$
a_{1 s}^{2}+a_{2 s}^{2}+2 a_{1 s} a_{2 s} \cos \left(\Delta \psi_{s}\right)=4 \frac{\varphi_{1}}{\varphi_{3}}
$$


One notes that there exist an infinity of steady-state points and that the convergence of the amplitude and phase depends essentially on the initial state of $x_{1}$ and $x_{2}$. Therefore, to get the same result between (15) and (30), one must initialize (30) with the appropriate values of initial amplitude and phase.

Figure 4 shows a simulation test of mutual synchronization with close frequencies phenomenon, the upper part are the outputs of (15) and lower part are the outputs approximated by (29) and (30).

\subsubsection{Mutual synchronization with multiple harmonics}

For the case where the frequency $\omega_{1}$ is close to $3 \omega_{2}$, the terms with frequencies $\omega_{1}$ and $3 \omega_{2}$ are used for $x_{1}$ approximation, and the terms with frequencies $\omega_{2}$ and $\left(2 \omega_{2}-\omega_{1}\right)$ are used for $x_{2}$ approximation. Therefore, one finds the result presented in following lemma

\section{Lemma 3}

Consider the case where the frequencies $\omega_{1}$ and $\omega_{2}$ satisfy

$$
\omega_{1} \approx 3 \omega_{2}
$$

the K-B approximation of (15) gives

$$
\begin{aligned}
& x_{i}=a_{i} \cos \left(\omega_{i} t+\theta_{i}\right),(i=1,2) \\
& \text { with } \\
& \left\{\begin{array}{c}
\frac{d a_{1}}{d t}=\varphi_{1}\left[\frac{a_{1}}{2}\left(1-\frac{\varphi_{3}}{\varphi_{1}}\left(\frac{a_{1}^{2}}{4}+\frac{a_{2}^{2}}{2}\right)\right)-\frac{\varphi_{3}}{\varphi_{1}} \frac{\omega_{2} a_{2}^{3}}{8 \omega_{1}} \cos (\Delta \psi)\right] \\
\frac{d a_{2}}{d t}=\varphi_{1}\left[\frac{a_{2}}{2}\left(1-\frac{\varphi_{3}}{\varphi_{1}}\left(\frac{a_{2}^{2}}{4}+\frac{a_{1}^{2}}{2}\right)\right)-\frac{a_{1} a_{2}^{2}}{8 \omega_{2}} \frac{\varphi_{3}}{\varphi_{1}}\left(\omega_{1}-2 \omega_{2}\right) \cos (\Delta \psi)\right], \\
\frac{d \theta_{1}}{d t}=\varphi_{3} \frac{\omega_{2} a_{2}^{3}}{8 \omega_{1} a_{1}} \sin (\Delta \psi), \\
\frac{d \theta_{2}}{d t}=-\varphi_{3} \frac{a_{1} a_{2}}{8 \omega_{2}}\left(\omega_{1}-2 \omega_{2}\right) \sin (\Delta \psi), \\
\text { where } \\
\Delta \psi=\psi_{1}-3 \psi_{2}=\left(\omega_{1}-3 \omega_{2}\right) t+\theta_{1}-3 \theta_{2} .
\end{array}\right.
\end{aligned}
$$

The proof of this lemma is given in appendix C. Let us find steady-state solution of (37). One stable steady-state point can be computed analytically from (37)

$$
a_{1 s}=2 \sqrt{\frac{\varphi_{1}}{\varphi_{3}}} \text { and } \quad a_{2 s}=0
$$

Others points which concern synchronization phenomenon need numerical solution of (37). Furthermore, one introduces

$$
\rho=\left|\frac{\omega_{1}-3 \omega_{2}}{\omega_{2}}\right| .
$$

For instance, in the case where $\omega_{1}=3 \omega_{2}=6 \pi \times 210$ (correspond to $\rho=0$ ), $\varphi_{1}=1.78 \times 10^{2}$ and $\varphi_{3}=1.24 \times 10^{7}$, the second steady state solution is given by

$$
a_{1 s}=2.25 \times 10^{-3}, a_{2 s}=8.09 \times 10^{-3} \text { and } \Delta \psi_{s}=\pi .
$$




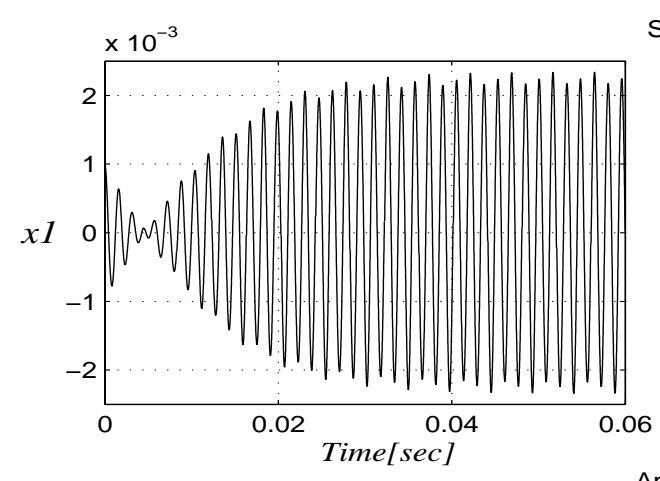

Simulation
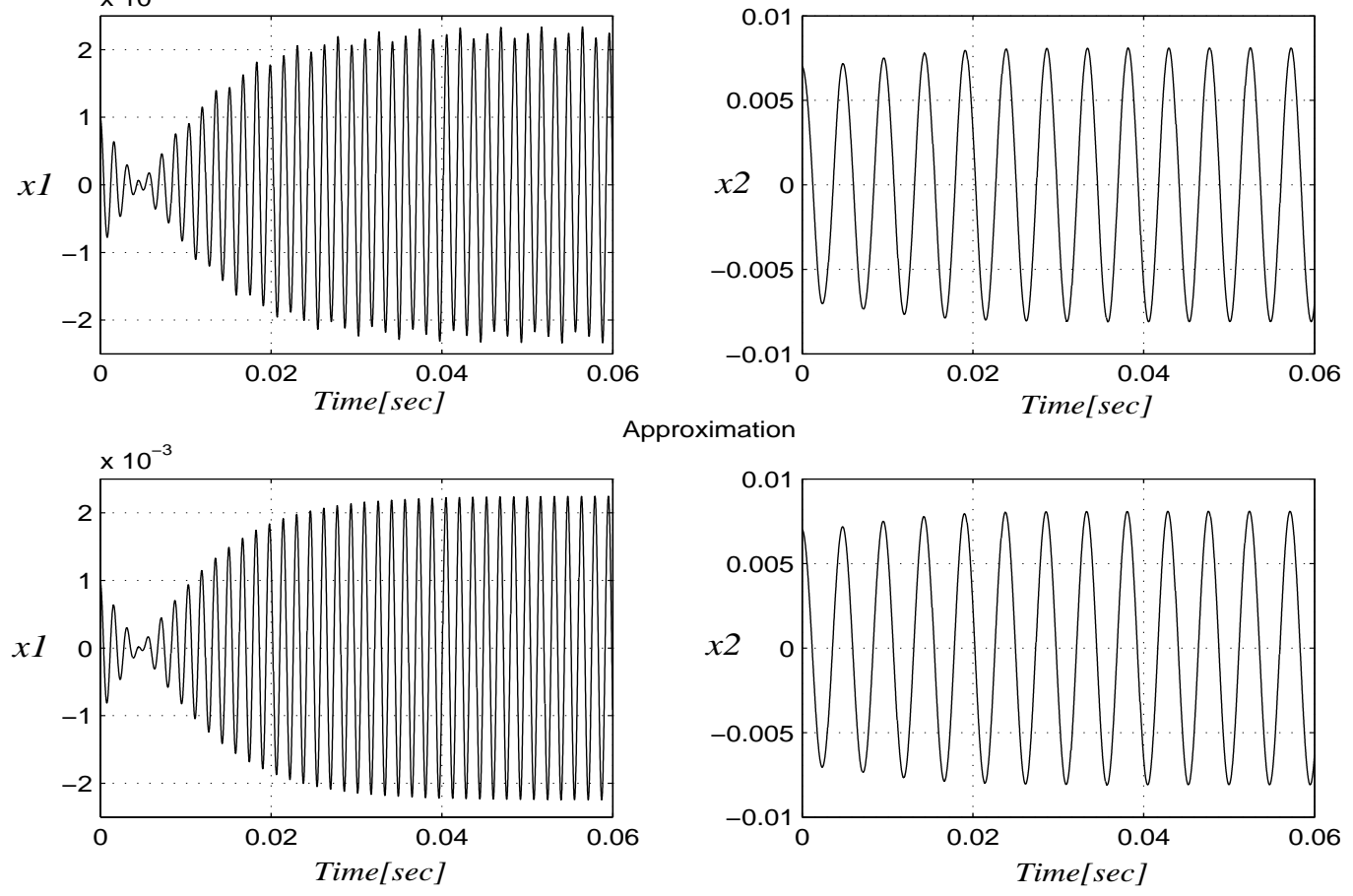

Approximation

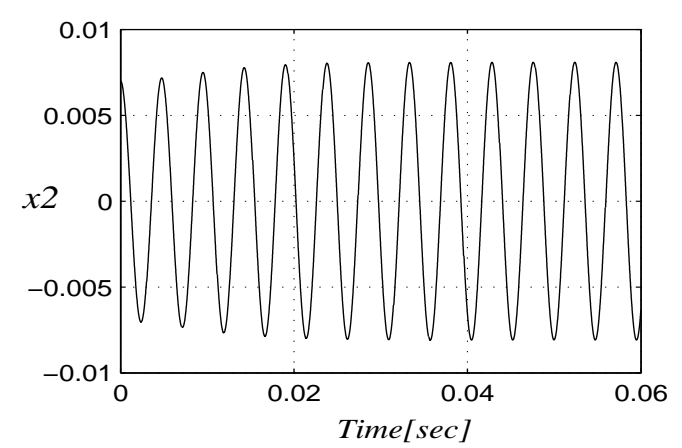

Figure 5: Simulation test with $\omega_{1}=3 \omega_{2}=6 \pi \times 210, \varphi_{1}=1.78 \times 10^{2}, \varphi_{3}=1.24 \times 10^{7}, a_{1}(0)=$. $10^{-3}, a_{2}(0)=6 \times 10^{-3}$ and $\theta_{1}(0)=\theta_{1}(0)=0$

For $\rho \neq 0$, it exists one other stable steady-state point which depend from the value of $p$ and $\omega_{2}$ (or $\omega_{1}$ ). It was noted that the amplitude $a_{1}$ decrease when $\rho$ increase.

From this, one can see that if $\omega_{1}$ is close to $3 \omega_{2}$ (respectively $\frac{\omega_{2}}{3}$ ), it is possible to have two phenomena depending on the initial condition. In the first phenomenon, the generator with frequency $\omega_{1}$ is excited and the other generator with frequency $\omega_{2}$ is quenched. In the second phenomenon, one has synchronization regime. By a synchronization regime is meant that the oscillation frequency of the second generator, which is equal to $\omega_{2}+\dot{\theta}_{2}$, is exactly a third of the oscillation frequency of the first generator, which is equal to $\omega_{1}+\dot{\theta}_{1}$. This phenomenon is illustrated by simulation test presented in Figure 5, the upper part are the outputs of (15) and lower part are the outputs approximated by (36) and (37).

\subsection{Summary of the analysis results}

We have identified three situations relating the proximity of the natural frequencies of the individual oscillators. From a practical point of view one can say that the system is characterized in steady state either by a single oscillating frequency (which corresponds to one of the resonance frequencies of the linear oscillators) or by stable simultaneous oscillations (which correspond to synchronized oscillations of both generators).

This single oscillation phenomena which is known as competitive quenching phenomena, occurs when the ratio of resonance frequencies of the two resonators are different from 3,1 and $\frac{1}{3}$, and occurrence of one of the frequencies 
(among the two) will depend upon the initial conditions.

Stable simultaneous oscillations with two distinct frequencies will occur only when $\omega_{1} \approx 3 \omega_{2}$ or $\omega_{2} \approx 3 \omega_{1}$ and the initial condition is sufficiently good so that both generators are excited.

\section{Two coupled generalized van der Pol equations driven by periodic force}

In order to complete the analysis of two coupled generalized van der Pol equations we deal in this section with nonautonomous case. We consider here the structure presented in (15) and introduce an additive force $v$ at input of linear resonators. This modification leads to following differential equations

$$
\left\{\begin{array}{c}
\frac{d^{2} x_{1}}{d t^{2}}+\omega_{1}^{2} x_{1}=\frac{d}{d t}\left(\varphi_{0}+\varphi_{1} y-\frac{\varphi_{3}}{3} y^{3}\right)+v \\
\frac{d^{2} x_{2}}{d t^{2}}+\omega_{2}^{2} x_{2}=\frac{d}{d t}\left(\varphi_{0}+\varphi_{1} y-\frac{\varphi_{3}}{3} y^{3}\right)+v \\
y=x_{1}+x_{2}
\end{array}\right.
$$

This structure will be analyzed for the case where the ratio of natural frequencies are different from 3,1 and $\frac{1}{3}$, and for periodic force

$$
v=B_{v} \cos \left(\omega_{v} t\right)
$$

where $B_{v}$ and $\omega_{v}$ are amplitude and radian frequency of periodic force, respectively. From system standpoint, this structure can be seen as a system driven by noise and the analysis allows estimation of noise effects. Generally, the outputs are affected differently depending on proximity between forced frequency and natural frequencies. Using K-B analysis, we will examine the cases where this input affects the dynamic characteristics and estimate the induced modifications (quench or excite oscillations). Let's use the following notation in the remaining of the paper

$$
\left\{\begin{aligned}
\triangle_{1} & =\left(\omega_{1}^{2}-\omega_{v}^{2}\right) \\
\triangle_{2} & =\left(\omega_{2}^{2}-\omega_{v}^{2}\right)
\end{aligned}\right.
$$

These parameters can be used to measure the distance between natural frequencies and the external frequency . Depending on frequency $\omega_{v}$, two situations are possible. In the first, we have an asynchronous situation which occurs when $\omega_{\nu}$ is different from natural frequencies. The second corresponds to a synchronous situation, which occurs when $\omega_{v}$ is close to one of the two natural frequencies.

\subsection{Asynchronous force}

Consider the case where the forced frequency is different form the both natural frequencies, which verified the condition (20). In this case, there is no interconnection effect between the three frequencies. For solving (42), we propose the following substitution

$$
x_{i}=z_{i}+\frac{B_{v}}{\triangle_{i}} \cos \left(\omega_{v} t\right), \quad(i=1,2)
$$


which leads to following deferential equations

$$
\begin{aligned}
& \left\{\begin{array}{l}
\frac{d^{2} z_{1}}{d t^{2}}+\omega_{1}^{2} z_{1}=f \\
\frac{d^{2} z_{2}}{d t^{2}}+\omega_{2}^{2} z_{2}=f
\end{array}\right. \\
& f=\varphi_{1}\left[1-\frac{\varphi_{3}}{\varphi_{1}}\left(z_{1}+z_{2}+\left(\frac{B_{v}}{\triangle_{1}}+\frac{B_{v}}{\triangle_{2}}\right) \cos \left(\omega_{v} t\right)\right)^{2}\right]\left(\frac{d z_{1}}{d t}+\frac{d z_{2}}{d t}-\left(\frac{B_{v}}{\triangle_{1}}+\frac{B_{v}}{\triangle_{2}}\right) \omega_{\nu} \sin \left(\omega_{v} t\right)\right) .
\end{aligned}
$$

With the new equations system, the periodic force have indirect effects on outputs $z_{1}$ and $z_{2}$ which can be measured using K-B approximation.

\section{Lemma 4}

Under conditions (20) and

$$
\left.\omega_{v} \not \approx \omega_{1}, \omega_{2}\right\}
$$

The solutions of (46) can be approached by

$$
\begin{aligned}
& z_{i}=a_{i} \cos \left(\omega_{i} t+\theta_{i}\right), \quad(i=1,2) \\
& \text { with } \\
& \left\{\begin{array}{l}
\frac{d a_{1}}{d t}=\frac{\varphi_{1} a_{1}}{2}\left(1-\frac{\varphi_{3}}{2 \varphi_{1}}\left(\frac{B_{v}}{\triangle_{1}}+\frac{B_{v}}{\triangle_{2}}\right)^{2}-\frac{\varphi_{3}}{\varphi_{1}}\left(\frac{a_{1}^{2}}{4}+\frac{a_{2}^{2}}{2}\right)\right), \\
\frac{d a_{2}}{d t}=\frac{\varphi_{1} a_{2}}{2}\left(1-\frac{\varphi_{3}}{2 \varphi_{1}}\left(\frac{B_{v}}{\triangle_{1}}+\frac{B_{v}}{\triangle_{2}}\right)^{2}-\frac{\varphi_{3}}{\varphi_{1}}\left(\frac{a_{2}^{2}}{4}+\frac{a_{1}^{2}}{2}\right)\right), \\
\frac{d \theta_{1}}{d t}=0 \\
\frac{d \theta_{2}}{d t}=0 .
\end{array}\right.
\end{aligned}
$$

The steps leading to this are presented in Appendix D. Comparing to autonomous case, one notes some modifications on amplitudes dynamic. The same amplitudes dynamic can be obtained in autonomous case by the substitution of $\varphi_{1}$ by $\varphi_{1}-\frac{\varphi_{3}}{2}\left(\frac{B_{v}}{\triangle_{1}}+\frac{B_{v}}{\triangle_{2}}\right)^{2}$, respectively. Consequently, this modification affects the value of steady amplitudes but does not change the two generators with competitive quenching behavior. Figure 6 shows a simulation test of (42) with asynchronous periodic force.

\subsection{Synchronous force}

Consider a periodic force with frequency $\omega_{v}$ close to one of the natural frequencies $\omega_{1}$ and $\omega_{2}$. Let's analyze the case where

$$
\omega_{v} \approx \omega_{2}
$$

For first resonator $x_{1}$, there is no interconnection effect with the forced frequency . Therefore, K-B approximation of $x_{1}$ gives the same result of asynchronous force case. However due to proximity of $\omega_{v}$ and $\omega_{2}$, an important interaction exists between the oscillations of $x_{2}$ and periodic force. This interaction leads to synchronization of $x_{2}$ by the periodic force that means an oscillation frequency in $x_{2}$ close to $\omega_{v}$. Therefore, we consider a solution of the form

$$
x_{2}=a_{2} \cos \left(\omega_{v} t+\theta_{2}\right)
$$



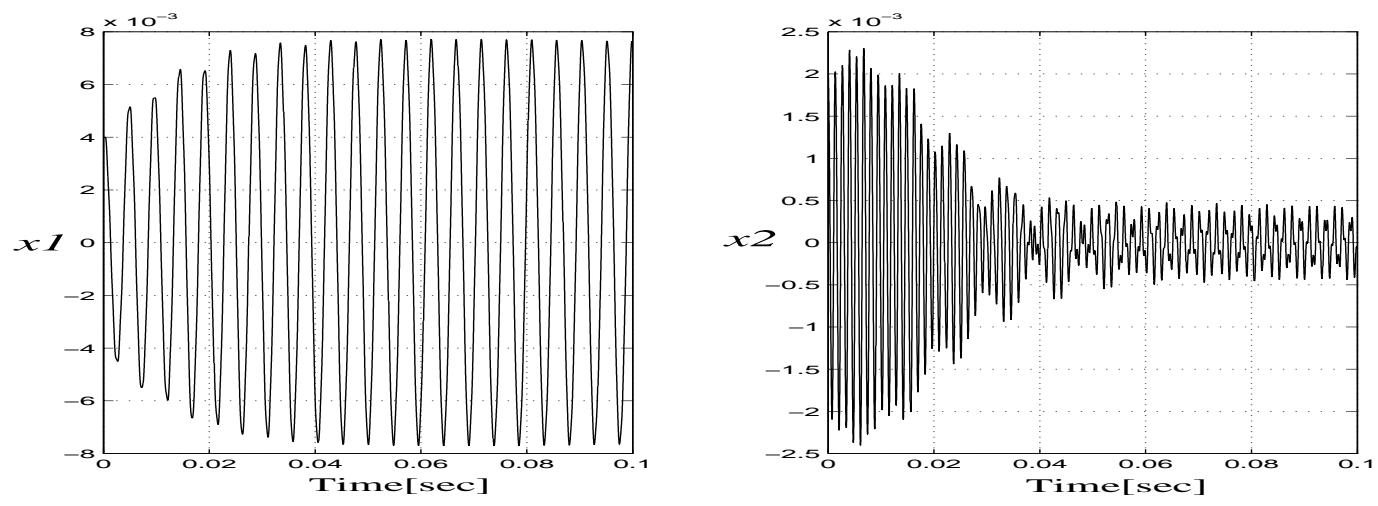

Figure 6: Simulation test of (42) with asynchronous periodic force, $\omega_{1}=2 \pi \times 210, \omega_{2}=2 \pi \times 740$, $\omega_{v}=2 \omega_{2}, B_{v}=10^{4}, \varphi_{0}=0.5, \varphi_{1}=1.78 \times 10^{2}$ and $\varphi_{3}=1.24 \times 10^{7}$.
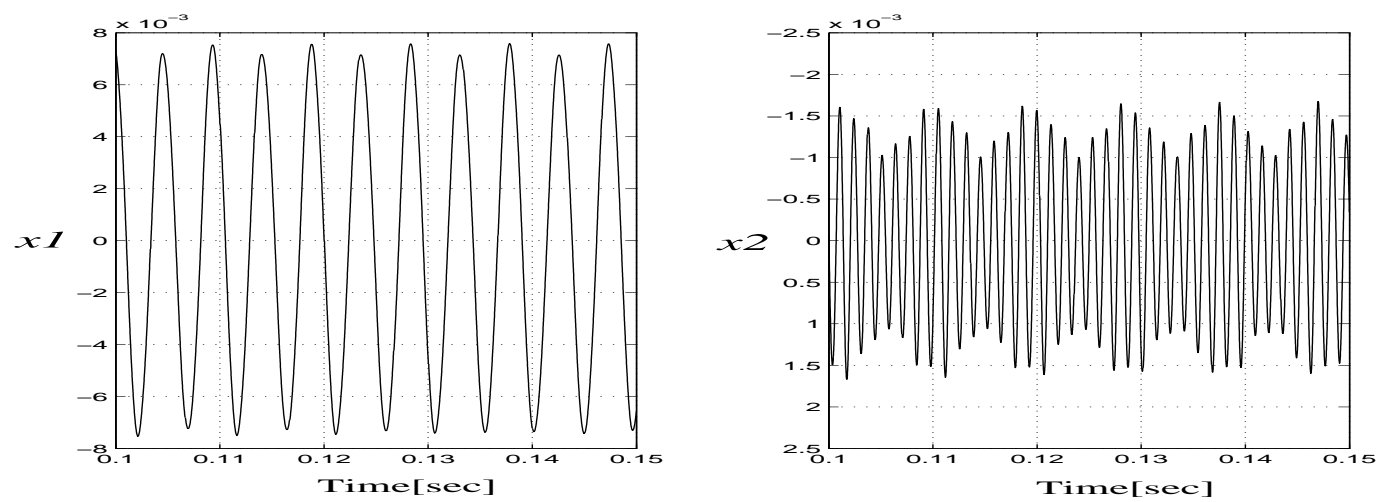

Figure 7: Simulation test of (42) with synchronous force, $\omega_{1}=2 \pi \times 210, \omega_{2}=\omega_{v}=2 \pi \times 740$, $B_{v}=1000, \varphi_{0}=0.5, \varphi_{1}=1.78 \times 10^{2}$ and $\varphi_{3}=1.24 \times 10^{7}$

\section{Lemma 5}

Under conditions (50), the outputs of system (42) can be approximated by

$$
\begin{aligned}
& \left\{\begin{array}{l}
x_{1}=a_{1} \cos \left(\omega_{1} t+\theta_{1}\right)+\frac{B_{v}}{\triangle_{1}} \cos \left(\omega_{v} t\right) \\
x_{2}=a_{2} \cos \left(\omega_{v} t+\theta_{2}\right)
\end{array}\right. \\
& \text { with } \\
& \left\{\begin{array}{l}
\frac{d a_{1}}{d t}=\frac{\varphi_{1} a_{1}}{2}\left(1-\frac{\varphi_{3}}{2 \varphi_{1}}\left(\frac{B_{v}}{\triangle_{1}}\right)^{2}-\frac{\varphi_{3}}{\varphi_{1}}\left(\frac{a_{1}^{2}}{4}+\frac{a_{2}^{2}}{2}\right)\right), \\
\frac{d a_{2}}{d t}=\frac{\varphi_{1} a_{2}}{2}\left(1-\frac{\varphi_{3}}{\varphi_{1}}\left(\frac{a_{2}^{2}}{4}+\frac{a_{1}^{2}}{2}\right)\right)-\frac{B_{v}}{2 \omega_{v}} \sin \left(\theta_{2}\right), \\
\frac{d \theta_{1}}{d t}=0, \\
\frac{d \theta_{2}}{d t}=-\frac{B_{v}}{2 \omega_{v} a_{2}} \cos \left(\theta_{2}\right)+\frac{\triangle_{2}}{2 \omega_{v}} .
\end{array}\right.
\end{aligned}
$$

The proof of this lemma is presented in Appendix E. Comparing with approximations obtained in the autonomous case, one observes coupling terms between the amplitude $a_{2}$ and the phase $\theta_{2}$, which implies the modification of steady-state solutions particulary on $a_{2}$. Tacking in account the differential equation of $\theta_{2}$, one concludes that amplitude $a_{2}$ can not be equal to zero. Consequently, the existence of synchronous periodic force leads to excitation of the 


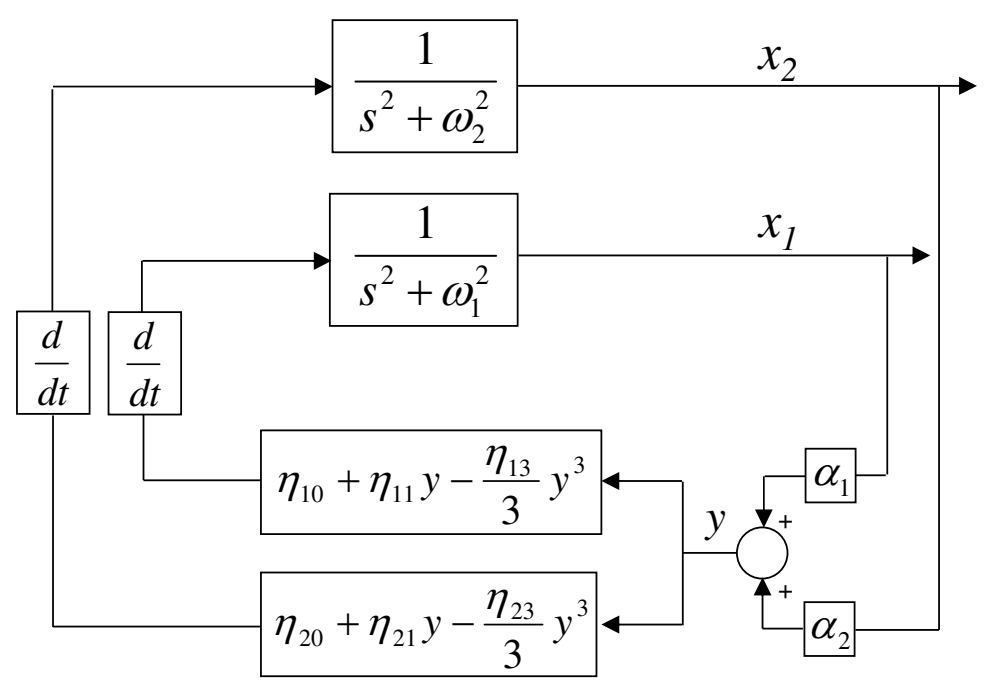

Figure 8: Two coupled resonators with different coupled terms

synchronized resonator.

In the high frequencies cases $\left(\omega_{v}>>1\right)$, the quantities $\frac{B_{v}}{2 \omega_{v}}$ and $\frac{B_{v}}{\triangle_{1}}$ are small and synchronous periodic force leads to small modifications on stable steady-state solutions which can be easily approximated. One stable steady-state solution can be approached by (26). The convergence of amplitude meant the excitation of second resonator oscillations and the quenching of first resonator oscillations. The second stable steady-state solution can be approached by the following expression (neglecting $a_{2}^{2}$ and $\frac{B_{v}^{2}}{\triangle_{1}^{2}}$ )

$$
a_{s 1} \approx 2 \sqrt{\frac{\varphi_{1}}{\varphi_{3}}}, a_{s 2} \approx \frac{B_{v}}{\omega_{\nu} \varphi_{1}} \frac{1}{\sqrt{1+\left(\frac{\triangle_{2}}{\omega_{\nu} \varphi_{1}}\right)^{2}}} \text { and } \theta_{s 2} \approx-\arctan \left(\frac{\omega_{\nu} \varphi_{1}}{\triangle_{2}}\right) \text {. }
$$

Consequently, on can concludes that through synchronous periodic force the system outputs are more affected than in the asynchronous cases. The oscillations of both resonators can coexist but with one small amplitude for high frequencies cases. Figure 7 shows the stationary part of a simulation test example in the case of synchronous periodic excitation.

\section{Two coupled resonators with different coupled terms}

The previous analysis shows that simultaneous oscillations with two distinct frequencies can not occurs when the ratio of resonance frequencies of the two resonators are different from 3,1 and $\frac{1}{3}$. In order to go further in the analysis of coupled oscillators, we define here another nonlinear coupled resonators structure

$$
\left\{\begin{array}{c}
\ddot{x}_{1}+\omega_{1}^{2} x_{1}=\frac{d}{d t}\left\{\eta_{10}+\eta_{11} y-\frac{\eta_{13}}{3} y^{3}\right\} \\
\ddot{x}_{2}+\omega_{2}^{2} x_{2}=\frac{d}{d t}\left\{\eta_{20}+\eta_{21} y-\frac{\eta_{23}}{3} y^{3}\right\} \\
y=\alpha_{1} x_{1}+\alpha_{2} x_{2} .
\end{array}\right.
$$


where $\eta_{10}, \eta_{20}, \eta_{11}, \eta_{21}, \eta_{13}$ and $\eta_{23}$ are arbitrary constants, $\alpha_{1}$ and $\alpha_{2}$ are arbitrary positive constants. The corresponding block diagram is depicted in Figure 8. Comparing to previous structure (15), the specification of this structure is that the van der Pol equations can have in general different nonlinear feedback characteristics (drift, slope, level of saturation).

\subsection{K-B analysis}

Consider the system (55) and the form (1) (with $\varepsilon=1$ ). Therefore, for the K-B approximation one may consider the following choice

$$
f_{i}=\frac{d}{d t}\left\{\eta_{i 0}+\eta_{i 1} y-\frac{\eta_{i 3}}{3} y^{3}\right\}=\eta_{i 1}\left\{1-\frac{\eta_{i 3}}{\eta_{i 1}}\left(\alpha_{1} x_{1}+\alpha_{2} x_{2}\right)^{2}\right\}\left(\alpha_{1} \dot{x}_{1}+\alpha_{2} \dot{x}_{2}\right),(i=1,2)
$$

Introducing (17) in (56) and after trigonometric simplifications, one obtains the expression

$$
\begin{aligned}
f_{i}= & -\omega_{1} \alpha_{1} \eta_{i 1} a_{1}\left[1-\frac{\eta_{i 3}}{\eta_{i 1}}\left(\frac{\left(\alpha_{1} a_{1}\right)^{2}}{4}+\frac{\left(\alpha_{2} a_{2}\right)^{2}}{2}\right)\right] \sin \left(\omega_{1} t+\theta_{1}\right)-\omega_{2} \alpha_{2} \eta_{i 1} a_{2}\left[1-\frac{\eta_{i 3}}{\eta_{i 1}}\left(\frac{\left(\alpha_{2} a_{2}\right)^{2}}{4}+\frac{\left(\alpha_{1} a_{1}\right)^{2}}{2}\right)\right. \\
& ] \sin \left(\omega_{2} t+\theta_{2}\right)+\frac{\eta_{i 3}}{4}\left\{\omega_{1}\left(\alpha_{1} a_{1}\right)^{3} \sin \left(3 \omega_{1} t+3 \theta_{1}\right)+\alpha_{1}^{2} \alpha_{2} a_{1}^{2} a_{2}\left[\left(2 \omega_{1}+\omega_{2}\right) \sin \left(\left(2 \omega_{1}+\omega_{2}\right) t+2 \theta_{1}+\theta_{2}\right)\right.\right. \\
& \left.+\left(2 \omega_{1}-\omega_{2}\right) \sin \left(\left(2 \omega_{1}-\omega_{2}\right) t+2 \theta_{1}-\theta_{2}\right)\right]+\alpha_{1} \alpha_{2}^{2} a_{1} a_{2}^{2}\left[\left(\omega_{1}+2 \omega_{2}\right) \sin \left(\left(\omega_{1}+2 \omega_{2}\right) t+\theta_{1}+2 \theta_{2}\right)\right. \\
& \left.\left.+\left(2 \omega_{2}-\omega_{1}\right) \sin \left(\left(2 \omega_{2}-\omega_{1}\right) t+2 \theta_{2}-\theta_{1}\right)\right]+\omega_{2}\left(\alpha_{2} a_{2}\right)^{3} \sin \left(3 \omega_{2} t+3 \theta_{2}\right)\right\} .
\end{aligned}
$$

from this expression one observes the existence of same frequency set $W$ defined in (19). Consequently, one gets the same classification presented in section 4.1. However, we limit our study here to case where condition (20) is satisfied. This is new and more practically interesting when compared with section 4 .

\section{Lemma 6}

Consider the condition (20) and the result (57). The application of $K-B$ approximation gives

$$
\begin{aligned}
& x_{i}=a_{i} \cos \left(\omega_{i} t+\theta_{i}\right),(i=1,2) \\
& \text { with } \\
& \left\{\begin{array}{l}
\dot{a}_{1}=\frac{\alpha_{1} \eta_{11}}{2} a_{1}\left[1-\frac{\eta_{13}}{\eta_{11}}\left(\frac{\left(\alpha_{1} a_{1}\right)^{2}}{4}+\frac{\left(\alpha_{2} a_{2}\right)^{2}}{2}\right)\right], \\
\dot{a}_{2}=\frac{\alpha_{2} \eta_{21}}{2} a_{2}\left[1-\frac{\eta_{23}}{\eta_{21}}\left(\frac{\left(\alpha_{2} a_{2}\right)^{2}}{4}+\frac{\left(\alpha_{1} a_{1}\right)^{2}}{2}\right)\right], \\
\dot{\theta}_{1}=0 \\
\dot{\theta}_{2}=0
\end{array}\right.
\end{aligned}
$$

By adopting the same procedure presented in Appendix A on function (57), one finds the result of lemma 6. From (59), one can see that the coupled parameters are $a_{1}$ and $a_{2}$. Therefore, the system dynamics depend essentially on the evolution of amplitudes $a_{1}$ and $a_{2}$. The analytical determination of equilibrium points $a_{s 1}$ and $a_{s 2}$ gives

$$
\begin{gathered}
a_{s 1}=0 \text { and } a_{s 2}=0, \\
a_{s 1}=\frac{2}{\alpha_{1}} \sqrt{\frac{\eta_{11}}{\eta_{13}}} \text { and } a_{s 2}=0, \\
a_{s 1}=0 \text { and } a_{s 2}=\frac{2}{\alpha_{2}} \sqrt{\frac{\eta_{21}}{\eta_{23}}}, \\
a_{s 1}=\frac{2}{\alpha_{1}} \sqrt{\frac{2 \eta_{21}}{3 \eta_{23}}-\frac{\eta_{11}}{3 \eta_{13}}} \text { and } a_{s 2}=\frac{2}{\alpha_{2}} \sqrt{\frac{2 \eta_{11}}{3 \eta_{13}}-\frac{\eta_{21}}{3 \eta_{23}}} .
\end{gathered}
$$


The stability of each equilibrium point leads to a particular regime. Consequently, one distinguishes five operation regimes, which will be elaborated and explained shortly :

1. Asymptotically stable system,

2. Two generators with competitive quenching,

3. One generator of van der Pol,

4. Simultaneous self-sustained oscillations,

5. Total instability.

For stability study one can apply the Lyapunov's indirect method, which consist to use the stability property of the linearized system around the equilibrium point. The computation of the general linearized matrix $M$ and characteristic polynomial $P(\lambda)$ leads to the following results

$$
\begin{gathered}
M=\left[\begin{array}{cc}
\frac{\alpha_{1} \eta_{11}}{2}-\frac{\alpha_{1} \eta_{13}}{2}\left(3 \frac{\left(\alpha_{1} a_{s 1}\right)^{2}}{4}+\frac{\left(\alpha_{2} a_{s 2}\right)^{2}}{2}\right) & -\frac{\eta_{13} \alpha_{1} \alpha_{2}^{2}}{2} a_{s 1} a_{s 2} \\
-\frac{\eta_{23} \alpha_{2} \alpha_{1}^{2}}{2} a_{s 1} a_{s 2} & \frac{\alpha_{2} \eta_{21}}{2}-\frac{\alpha_{2} \eta_{23}}{2}\left(3 \frac{\left(\alpha_{2} a_{s 2}\right)^{2}}{4}+\frac{\left(\alpha_{1} a_{s 1}\right)^{2}}{2}\right)
\end{array}\right] \\
P(\lambda)=\lambda^{2}-\frac{1}{2}\left\{\alpha_{1} \eta_{11}+\alpha_{2} \eta_{21}-\alpha_{1} \eta_{13}\left(\frac{3\left(\alpha_{1} a_{s 1}\right)^{2}}{4}+\frac{\left(\alpha_{2} a_{s 2}\right)^{2}}{2}\right)-\alpha_{2} \eta_{23}\left(\frac{3\left(\alpha_{2} a_{s 2}\right)^{2}}{4}+\frac{\left(\alpha_{1} a_{s 1}\right)^{2}}{2}\right)\right\} \lambda \\
+\frac{\alpha_{1} \alpha_{2}}{4}\left[\eta_{11}-\eta_{13}\left(\frac{3\left(\alpha_{1} a_{s 1}\right)^{2}}{4}+\frac{\left(\alpha_{2} a_{s 2}\right)^{2}}{2}\right)\right]\left[\eta_{21}-\eta_{23}\left(\frac{3\left(\alpha_{2} a_{s 2}\right)^{2}}{4}+\frac{\left(\alpha_{1} a_{s 1}\right)^{2}}{2}\right)\right]-\frac{\eta_{13} \eta_{23}\left(\alpha_{1} \alpha_{2}\right)^{3}\left(a_{s 1} a_{s 2}\right)^{2}}{4} .
\end{gathered}
$$

The characteristic polynomial obtained is a second order polynomial. Therefore, the stability can be verified by testing the sign of polynomial coefficients (should be positive).

\subsubsection{Asymptotic stable system}

The system is asymptotically stable around the origin, if and only if the equilibrium point (60) is asymptotically stable. Introducing (60) in the general characteristic polynomial (65), one obtains

$$
P(\lambda)=\lambda^{2}-\frac{1}{2}\left\{\alpha_{1} \eta_{11}+\alpha_{2} \eta_{21}\right\} \lambda+\frac{\alpha_{1} \eta_{11} \alpha_{2} \eta_{21}}{4},
$$

which has two stable zeros if the following conditions are satisfied

$$
\left\{\begin{array} { l } 
{ - \{ \alpha _ { 1 } \eta _ { 1 1 } + \alpha _ { 2 } \eta _ { 2 1 } \} > 0 } \\
{ \alpha _ { 1 } \eta _ { 1 1 } \alpha _ { 2 } \eta _ { 2 1 } > 0 }
\end{array} \Longleftrightarrow \left\{\begin{array}{l}
\eta_{11}<0 \\
\eta_{21}<0
\end{array}\right.\right.
$$

Provided (67) is satisfied and the initial states are close to the origin, the amplitudes of both oscillations converge to the equilibrium point (60). The satisfaction of conditions (67) imply positives damping effect about the origin. Figure 9 shows an example of a simulation test of the system (55), where the conditions (67) are satisfied. 

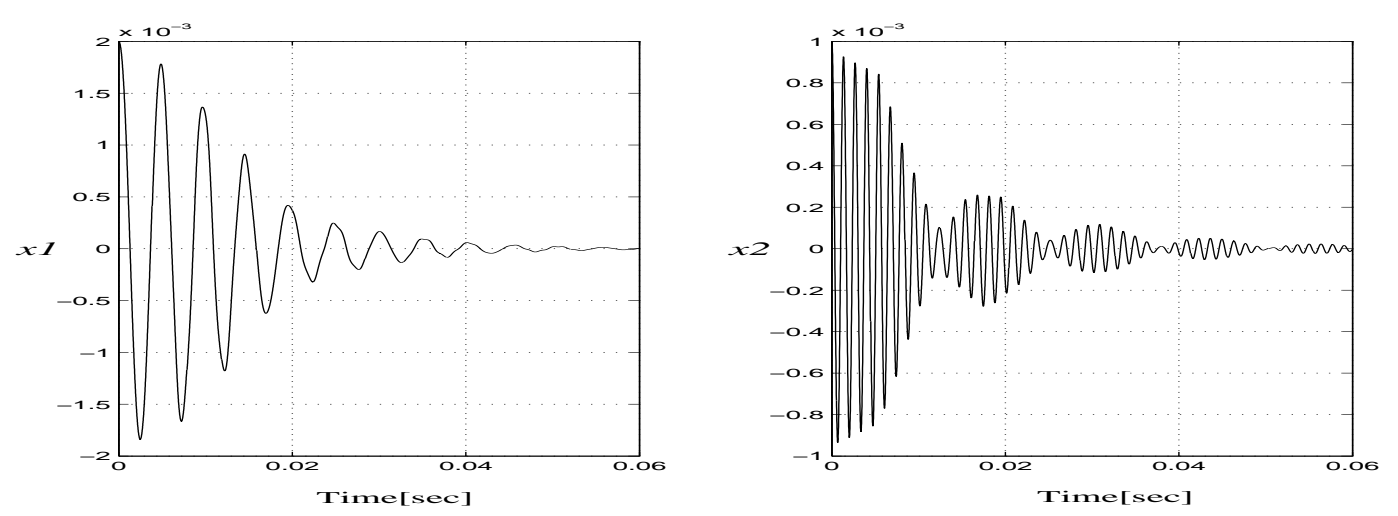

Figure 9: Asymptotic stable system - Simulation test with $\alpha_{1}=\omega_{1}=2 \pi \times 210, \alpha_{2}=\omega_{2}=2 \pi \times$ $740, \eta_{10}=\eta_{20}=0.5, \eta_{11}=-0.142, \eta_{21}=-8.5 \times 10^{-2}, \eta_{13}=-5 \times 10^{-3}$ and $\eta_{23}=$ $-3 \times 10^{-3}$.

\subsubsection{Two generators with competitive quenching}

The two generators with competitive quenching regime occurs when both equilibrium points (61) and (62) are locally stable. Substituting (61) into (65), one gets

$$
P(\lambda)=\lambda^{2}+\frac{1}{2}\left\{2 \alpha_{1} \eta_{11}+2 \alpha_{2} \eta_{23} \frac{\eta_{11}}{\eta_{13}}-\alpha_{2} \eta_{21}\right\} \lambda+\frac{\alpha_{1} \alpha_{2} \eta_{11}}{2}\left\{2 \eta_{23} \frac{\eta_{11}}{\eta_{13}}-\eta_{21}\right\}
$$

The local stability of (61) is satisfied if and only if

$$
\left\{\begin{array} { l } 
{ 2 \alpha _ { 1 } \eta _ { 1 1 } + 2 \alpha _ { 2 } \eta _ { 2 3 } \frac { \eta _ { 1 1 } } { \eta _ { 1 3 } } - \alpha _ { 2 } \eta _ { 2 1 } > 0 } \\
{ \alpha _ { 1 } \alpha _ { 2 } \eta _ { 1 1 } \{ 2 \eta _ { 2 3 } \frac { \eta _ { 1 1 } } { \eta _ { 1 3 } } - \eta _ { 2 1 } \} > 0 }
\end{array} \Longleftrightarrow \left\{\begin{array}{l}
\eta_{11}>0 \\
2 \eta_{23} \frac{\eta_{11}}{\eta_{13}}-\eta_{21}>0
\end{array}\right.\right.
$$

By symmetry, for the equilibrium point (62) one finds the same conditions.

$$
\left\{\begin{array}{l}
2 \eta_{13} \frac{\eta_{21}}{\eta_{23}}-\eta_{11}>0 \\
\eta_{21}>0
\end{array}\right.
$$

Provided that the conditions (69) and (70) are satisfied, the amplitudes of $x_{1}$ and $x_{2}$ converge to one of both possible equilibrium points (61) and (62). Depending on the initial states, one of the generators is excited, while the oscillations of the other generator are entirely quenched. This situation is more general than situation encountered in two coupled generalized van der Pol equations case described in section 4.1.1.

\subsubsection{One van der Pol generator}

This regime occurs when one of the equilibrium points (61) and (62) is stable and the other is unstable. For example, if conditions (69) are satisfied and at least one condition of (70) is not satisfied, this implies that only equilibrium point (61) is stable and for any initial states the generator $x_{1}$ is excited and the generated $x_{2}$ is quenched (the converse is correct). The main difference between this regime and two generators with competitive quenching regime, is that the excitation of one of the two generators does not depend only on initial condition, but depend also on satisfying of stability conditions, and than the system behaves as one equation of van der Pol type. 


\subsubsection{Simultaneous self-sustained oscillations}

Simultaneous and persistent oscillation of both resonators occurs when the equilibrium point (63) is stable. Introducing (63) in (65), one obtains

$$
P(\lambda)=\lambda^{2}+\frac{1}{3}\left\{\alpha_{1} \eta_{13}\left[\frac{2 \eta_{21}}{\eta_{23}}-\frac{\eta_{11}}{\eta_{13}}\right]+\alpha_{2} \eta_{23}\left[\frac{2 \eta_{11}}{\eta_{13}}-\frac{\eta_{21}}{\eta_{23}}\right]\right\} \lambda-\frac{\alpha_{1} \alpha_{2} \eta_{13} \eta_{23}}{3}\left[\frac{2 \eta_{21}}{\eta_{23}}-\frac{\eta_{11}}{\eta_{13}}\right]\left[\frac{2 \eta_{11}}{\eta_{13}}-\frac{\eta_{21}}{\eta_{23}}\right]
$$

The equilibrium point (63) is locally stable if and only if

$$
\left\{\begin{array}{l}
\alpha_{1} \eta_{13}\left[\frac{2 \eta_{21}}{\eta_{23}}-\frac{\eta_{11}}{\eta_{13}}\right]+\alpha_{2} \eta_{23}\left[\frac{2 \eta_{11}}{\eta_{13}}-\frac{\eta_{21}}{\eta_{23}}\right]>0 \\
\alpha_{1} \alpha_{2} \eta_{13} \eta_{23}\left[\frac{2 \eta_{21}}{\eta_{23}}-\frac{\eta_{11}}{\eta_{13}}\right]\left[\frac{2 \eta_{11}}{\eta_{13}}-\frac{\eta_{21}}{\eta_{23}}\right]<0
\end{array}\right.
$$

Knowing that for the existence of equilibrium point (63), the following conditions must be satisfied

$$
\left\{\begin{array} { l } 
{ \frac { 2 \eta _ { 1 1 } } { \eta _ { 1 3 } } - \frac { \eta _ { 2 1 } } { \eta _ { 2 3 } } > 0 } \\
{ \frac { 2 \eta _ { 2 1 } } { \eta _ { 2 3 } } - \frac { \eta _ { 1 1 } } { \eta _ { 1 3 } } > 0 }
\end{array} \Longrightarrow \left\{\begin{array}{l}
\alpha_{1} \eta_{13}\left[\frac{2 \eta_{21}}{\eta_{23}}-\frac{\eta_{11}}{\eta_{13}}\right]+\alpha_{2} \eta_{23}\left[\frac{2 \eta_{11}}{\eta_{13}}-\frac{\eta_{21}}{\eta_{23}}\right]>0 \\
\eta_{13} \eta_{23}<0
\end{array}\right.\right.
$$

By satisfying (73), it is possible to have simultaneous self-sustained oscillations. The amplitudes of $x_{1}$ and $x_{2}$ converge to the equilibrium point (63). By self-sustained oscillations it is meant that both oscillators are excited without synchronization. Figure 10 shows the stationary part of a simulation test example of simultaneous self-sustained oscillatory regime, where the upper part shows the simulated outputs (55) and the lower part shows the approximated outputs (58) and (59).

\subsubsection{Total instability}

When conditions (67), (69), (70) and (73) are not satisfied, there does not exist a stable equilibrium point. Therefore, there is no stable limit cycle and the amplitudes of both oscillations diverge. By total instability it is meant that for any non-equilibrium initial state, the state of the system diverges.

\subsection{Summary of the analysis results}

The results demonstrate the existence of five operation regimes when the ratio of natural frequencies is different from 1,3 and $\frac{1}{3}$. The occurrence of each operation regime depends on the satisfaction of some conditions. The simulations confirm the quality of estimated amplitudes using K-B approximation.

The absence of self excited oscillations in both resonators occurs when $\eta_{11}$ and $\eta_{21}$ are positive. Two generators with competitive quenching regime occurs when both conditions (69) and (70) are satisfied, which is possible if $\eta_{11}$ and $\eta_{21}$ are negative. The excitation of one of both resonator depend on initial amplitudes. The excitation of only one resonator independently on initial amplitudes occurs if one of these conditions is satisfied while the other is not satisfied. Stable simultaneous oscillations with two distinct frequencies and without synchronization which is known as Simultaneous self-sustained oscillations operates when conditions (73) are verified, which implies $\eta_{13}$ and $\eta_{23}$ with different signs. The divergence of oscillations occurs when none of the conditions cited previously is satisfied. 

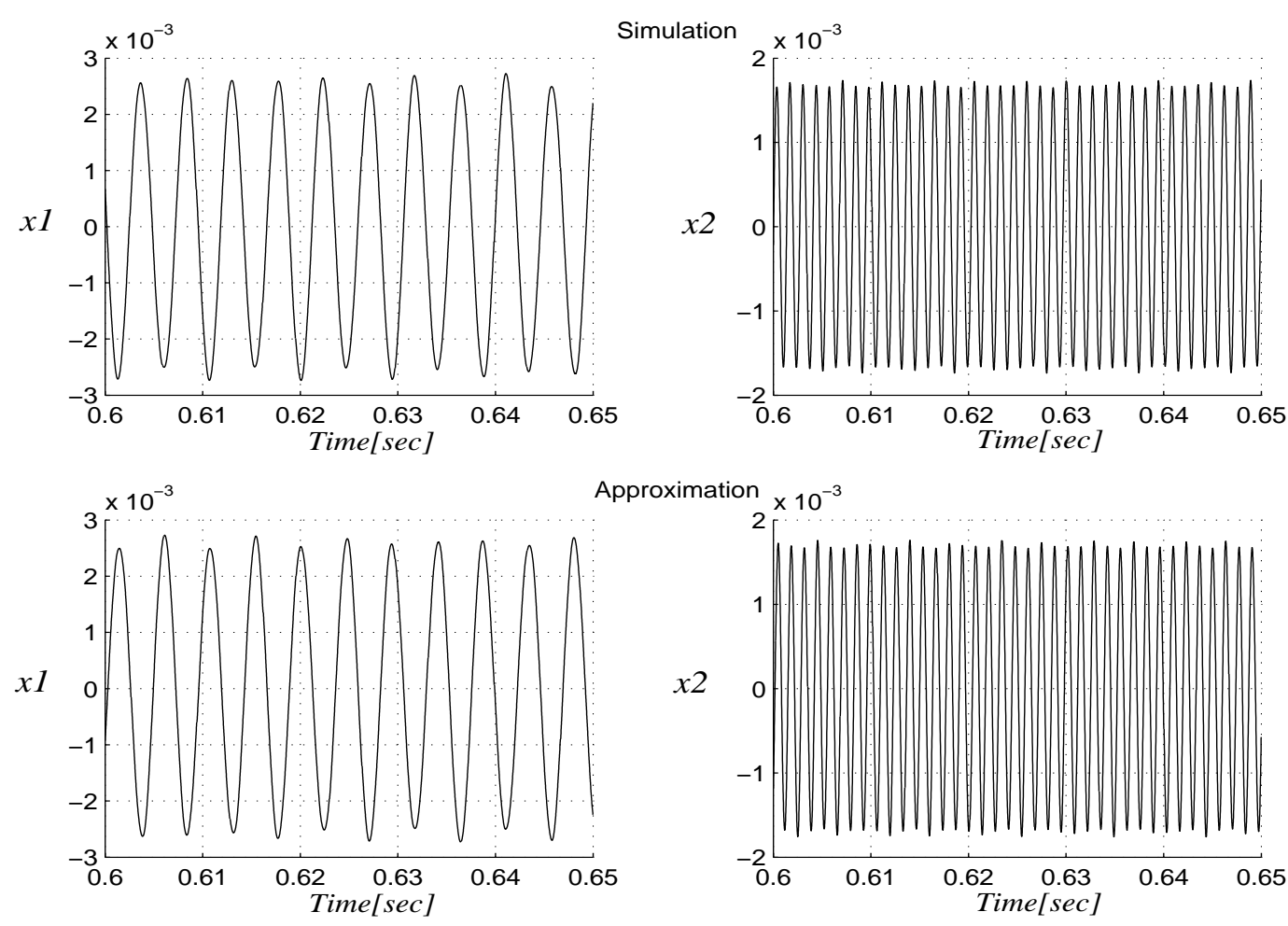

Approximation $2 \times 10^{-3}$

Figure 10: Simultaneous self-sustained oscillations - Simulation test with $\alpha_{1}=\omega_{1}=2 \pi \times 210$, $\alpha_{2}=\omega_{2}=2 \pi \times 740, \eta_{10}=\eta_{20}=0.5, \eta_{11}=-7.4 \times 10^{-2}, \eta_{21}=6.3 \times 10^{-2}, \eta_{13}=$ $-2 \times 10^{-3}$ and $\eta_{23}=3 \times 10^{-3}$.

\subsection{Equivalence with a combustion instability model}

Combustion instabilities phenomena are without doubt very important in practice [1]. These phenomena are highly nonlinear phenomena, sometimes characterized by coexistence of oscillations at several distinct non-harmonic frequencies that makes the modelling of such phenomena extremely difficult. In order to explain the coexistence of two non-harmonic modes in combustion instabilities, an attractive model has been proposed in [5]. The model has two linear coupled resonator. The feedback path includes a nonlinear static characteristic, a delay and a filter. The model is represented in Figure 11. The equations associated with this model are:

$$
\left\{\begin{array}{l}
\ddot{x}_{1}+\omega_{1}^{2} x_{1}=\frac{d}{d t} L P F\left\{\varphi_{v 0}+\varphi_{v 1} \dot{p}_{\tau}-\frac{\varphi_{v 3}}{3} \dot{p}_{\tau}^{3}\right\}, \\
\ddot{x}_{2}+\omega_{2}^{2} x_{2}=\frac{d}{d t} L P F\left\{\varphi_{v 0}+\varphi_{v 1} \dot{p}_{\tau}-\frac{\varphi_{v 3}}{3} \dot{p}_{\tau}^{3}\right\}
\end{array}\right.
$$

where $\varphi_{v 0}$ is an arbitrary constant, $\varphi_{v 1}$ and $\varphi_{v 3}$ are arbitrary negative constants, $\tau$ is a transport time delay from nozzle to flame surface, $L P F$ is a transfer operator of the low pass filter, $p=x_{1}+x_{2}$ is the downstream pressure perturbation at the burning plane and $\dot{p}_{\tau}$ is the output of the delay-plus-differentiator block.

This model has been successfully analyzed using K-B method and two realistic assumptions on delay and filtering [5]. The analysis reveals for the case where the ratio of natural frequencies is different from 1,3 and $\frac{1}{3}$, that the 


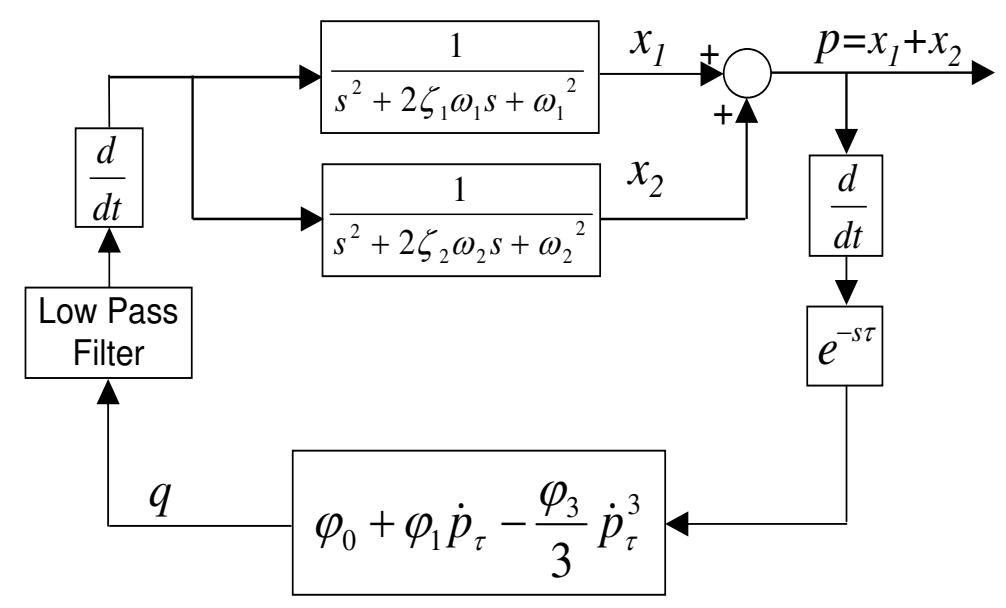

Figure 11: A combustion instability model.

amplitudes of oscillations can be approached by

$$
\left\{\begin{array}{l}
\dot{a}_{1}=\frac{\omega_{1} \varphi_{v 1} G\left(\omega_{1}\right) \sin \left[\omega_{1} \tau+\phi\left(\omega_{1}\right)\right]}{2} a_{1}\left[1-\frac{\varphi_{v 3}}{\varphi_{v 1}}\left(\frac{\left(\omega_{1} a_{1}\right)^{2}}{4}+\frac{\left(\omega_{2} a_{2}\right)^{2}}{2}\right)\right], \\
\dot{a}_{2}=\frac{\omega_{2} \varphi_{v 1} G\left(\omega_{2}\right) \sin \left[\omega_{2} \tau+\phi\left(\omega_{2}\right)\right]}{2} a_{2}\left[1-\frac{\varphi_{v 3}}{\varphi_{v 1}}\left(\frac{\left(\omega_{2} a_{2}\right)^{2}}{4}+\frac{\left(\omega_{1} a_{1}\right)^{2}}{2}\right)\right] .
\end{array}\right.
$$

where $G\left(\omega_{i}\right)$ and $\phi\left(\omega_{i}\right)(i=1,2)$ are the gain and the phase at frequency $\omega_{i}$ introduced by the low pass filter. Equation (75) corresponds in fact to equation (59) if we consider the following substitutions

$$
\left\{\begin{array}{lll}
\alpha_{1}=\omega_{1}, & \eta_{11}=\varphi_{v 1} G\left(\omega_{1}\right) \sin \left[\omega_{1} \tau+\phi\left(\omega_{1}\right)\right], & \eta_{13}=\varphi_{v 3} G\left(\omega_{1}\right) \sin \left[\omega_{1} \tau+\phi\left(\omega_{1}\right)\right], \\
\alpha_{2}=\omega_{2}, & \eta_{21}=\varphi_{v 1} G\left(\omega_{2}\right) \sin \left[\omega_{2} \tau+\phi\left(\omega_{2}\right)\right], & \eta_{23}=\varphi_{v 3} G\left(\omega_{2}\right) \sin \left[\omega_{2} \tau+\phi\left(\omega_{2}\right)\right],
\end{array}\right.
$$

Therefore, the model (74) can be represented by structure (55) and the coexistence of two non-harmonic modes occurs in the case when the conditions (73) are verified.

Comparing now Figure 11 with Figure 8 one can conclude that the presence of the delay in the feedback common path of the combustion instability model is equivalent to having two coupled resonators each with a different feedback path but without delay when the natural frequencies are different from 1,3 and $\frac{1}{3}$.

\section{Conclusion}

Simultaneous oscillations of distinct frequencies are encountered in a number of real systems subject to self-excitation of oscillations. The prototype model structures proposed in this paper can display such behavior. One of the proposed model structure can feature co-existence of two non harmonic oscillations. These models have been analyzed using the Krylov-Bogoliubov method and conditions for the occurrence of various operation regimes have been established.

The results provided are generic in the sense that if a model can be equivalently transformed to one of the proposed forms, its properties will result straightforwardly from the results given in the paper. This is illustrated for the case of a combustion instability model. 


\section{Appendix}

\section{A K-B approximation for two generators with competitive quenching case of struc- ture (15)}

Let's compute the K-B approximation of $x_{1}$ when condition (20) is verified. In this case, we have the following propriety

$$
\omega_{1} \not \approx\left\{\omega_{2}, 3 \omega_{1}, 3 \omega_{2}, 2 \omega_{1}+\omega_{2}, \omega_{1}+2 \omega_{2},\left|2 \omega_{1}-\omega_{2}\right|,\left|2 \omega_{2}-\omega_{1}\right|\right\} .
$$

Consequently, one can consider that (18) is exactly in the form (5) with

$$
\begin{aligned}
& H_{11}=-\omega_{1} \varphi_{1} a_{1}\left(1-\frac{\varphi_{3}}{\varphi_{1}}\left(\frac{a_{1}^{2}}{4}+\frac{a_{2}^{2}}{2}\right)\right), \\
& G_{11}=0 .
\end{aligned}
$$

Using (78) and (3), one finds

$$
\begin{aligned}
& x_{1}=a_{1} \cos \left(\omega_{1} t+\theta_{1}\right) \\
& \text { with } \\
& \left\{\begin{array}{l}
\frac{d a_{1}}{d t}=\varphi_{1} \frac{a_{1}}{2}\left(1-\frac{\varphi_{3}}{\varphi_{1}}\left(\frac{a_{1}^{2}}{4}+\frac{a_{2}^{2}}{2}\right)\right), \\
\frac{d \theta_{1}}{d t}=0 .
\end{array}\right.
\end{aligned}
$$

Symmetrically, by adopting the same procedure for $x_{2}$, one obtains

$$
\begin{aligned}
& x_{2}=a_{2} \cos \left(\omega_{2} t+\theta_{2}\right) \\
& \text { with } \\
& \left\{\begin{array}{l}
\frac{d a_{2}}{d t}=\varphi_{1} \frac{a_{2}}{2}\left(1-\frac{\varphi_{3}}{\varphi_{1}}\left(\frac{a_{2}^{2}}{4}+\frac{a_{1}^{2}}{2}\right)\right), \\
\frac{d \theta_{2}}{d t}=0 .
\end{array}\right.
\end{aligned}
$$

Assembling (2), (80) and (82), yielding at the end the result of lemma 1.

\section{B K-B approximation for mutual synchronization with close frequencies case}

Let's compute the K-B approximation of $x_{1}$ when the frequencies $\omega_{1}$ and $\omega_{2}$ are close. By using the notation (31), one has the relations

$$
\left\{\begin{array}{l}
\omega_{2} t+\theta_{2}=\omega_{1} t+\theta_{1}-\Delta \psi \\
\left(2 \omega_{1}-\omega_{2}\right) t+2 \theta_{1}-\theta_{2}=\omega_{1} t+\theta_{1}+\Delta \psi \\
\left(2 \omega_{2}-\omega_{1}\right) t+2 \theta_{2}-\theta_{1}=\omega_{1} t+\theta_{1}-2 \Delta \psi
\end{array}\right.
$$


Taking account properties (27), (28) and (83), the function (18) can be set in the form (5) as follows

$$
\begin{aligned}
f= & \varphi_{1}\left\{-\omega_{1} a_{1}\left[1-\frac{\varphi_{3}}{\varphi_{1}}\left(\frac{a_{1}^{2}}{4}+\frac{a_{2}^{2}}{2}\right)\right] \sin \left(\omega_{1} t+\theta_{1}\right)-\omega_{2} a_{2}\left[1-\frac{\varphi_{3}}{\varphi_{1}}\left(\frac{a_{2}^{2}}{4}+\frac{a_{1}^{2}}{2}\right)\right] \sin \left(\omega_{1} t+\theta_{1}-\Delta \psi\right)\right. \\
& \left.+\frac{\varphi_{3}}{\varphi_{1}}\left[\frac{\left(2 \omega_{1}-\omega_{2}\right) a_{1}^{2} a_{2}}{4} \sin \left(\omega_{1} t+\theta_{1}+\Delta \psi\right)+\frac{\left(2 \omega_{2}-\omega_{1}\right) a_{2}^{2} a_{1}}{4} \sin \left(\omega_{1} t+\theta_{1}-2 \Delta \psi\right)\right]\right\} \\
& +\sum_{\omega_{1}}^{4} \nsucc \omega_{\ell}\left(H_{\ell 1} \sin \left(\omega_{\ell} t+\theta_{\ell}\right)+G_{\ell 1} \cos \left(\omega_{\ell} t+\theta_{\ell}\right)\right) . \\
= & -\varphi_{1}\left\{\omega_{1} a_{1}\left[1-\frac{\varphi_{3}}{\varphi_{1}}\left(\frac{a_{1}^{2}}{4}+\frac{a_{2}^{2}}{2}\right)\right]+\left[\omega_{2} a_{2}\left(1-\frac{\varphi_{3}}{\varphi_{1}} \frac{a_{1}^{2}+a_{2}^{2}}{4}\right)-\frac{\omega_{1} \varphi_{3} a_{2} a_{1}^{2}}{2 \varphi_{1}}\right] \cos (\Delta \psi)\right. \\
& \left.+\frac{\varphi_{3}\left(\omega_{1}-2 \omega_{2}\right) a_{2}^{2} a_{1}}{4 \varphi_{1}} \cos (2 \Delta \psi)\right\} \sin \left(\omega_{1} t+\theta_{1}\right)+\varphi_{1}\left\{\left[\omega_{2} a_{2}\left(1-\frac{\varphi_{3}}{\varphi_{1}}\left(\frac{a_{2}^{2}}{4}+\frac{a_{1}^{2}}{2}\right)\right)+\frac{\varphi_{3}\left(2 \omega_{1}-\omega_{2}\right) a_{1}^{2} a_{2}}{4 \varphi_{1}}\right] \sin (\Delta \psi)\right. \\
& \left.-\frac{\varphi_{3}\left(2 \omega_{2}-\omega_{1}\right) a_{2}^{2} a_{1}}{4 \varphi_{1}} \sin (2 \Delta \psi)\right\} \cos \left(\omega_{1} t+\theta_{1}\right)+\sum_{\omega_{1}}^{4} \not \omega_{\ell}\left(H_{\ell 1} \sin \left(\omega_{\ell} t+\theta_{\ell}\right)+G_{\ell 1} \cos \left(\omega_{\ell} t+\theta_{\ell}\right)\right) .
\end{aligned}
$$

From which one can deduce

$$
\begin{aligned}
& \left.H_{11}=-\varphi_{1}\left\{\omega_{1} a_{1}\left[1-\frac{\varphi_{3}}{\varphi_{1}}\left(\frac{a_{1}^{2}}{4}+\frac{a_{2}^{2}}{2}\right)\right]+\left[\omega_{2} a_{2}\left(1-\frac{\varphi_{3} a_{1}^{2}+a_{2}^{2}}{4}\right)-\frac{\omega_{1} \varphi_{3} a_{2} a_{1}^{2}}{2 \varphi_{1}}\right] \cos (\Delta \psi)+\frac{\varphi_{3}\left(\omega_{1}-2 \omega_{2}\right) a_{2}^{2} a_{1}}{4 \varphi_{1}} \cos (2 \Delta \psi)\right\}_{(\dot{8}}\right) \\
& G_{11}=\varphi_{1}\left\{\left[\omega_{2} a_{2}\left(1-\frac{\varphi_{3}}{\varphi_{1}}\left(\frac{a_{2}^{2}}{4}+\frac{a_{1}^{2}}{2}\right)\right)+\frac{\varphi_{3}\left(2 \omega_{1}-\omega_{2}\right) a_{1}^{2} a_{2}}{4 \varphi_{1}}\right] \sin (\Delta \psi)-\frac{\varphi_{3}\left(2 \omega_{2}-\omega_{1}\right) a_{2}^{2} a_{1}}{4 \varphi_{1}} \sin (2 \Delta \psi)\right\} .
\end{aligned}
$$

Using (84) and (3), one finds

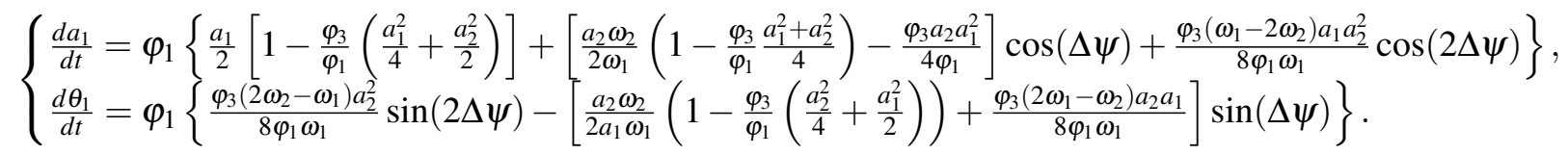

Symmetrically, by adopting the same procedure for $x_{2}$, one obtains

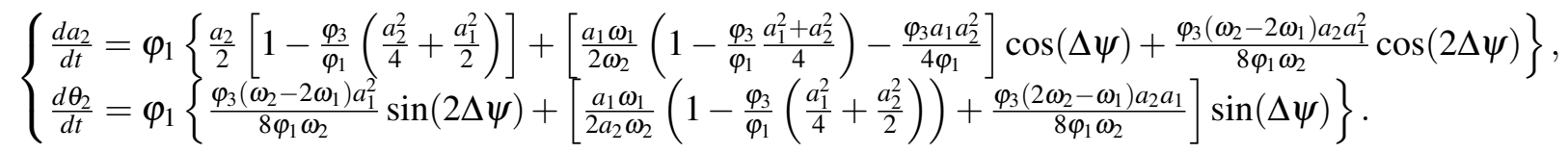

Assembling (85) and (86), yields at the end the equations system (30).

\section{K-B approximation for mutual synchronization with multiple harmonics case}

Let's compute the K-B approximation of $x_{1}$ when

$$
\begin{aligned}
& \omega_{1} \approx\left\{3 \omega_{2}\right\}, \\
& \Rightarrow \omega_{1} \not \approx\left\{\omega_{2}, 3 \omega_{1}, 2 \omega_{1}+\omega_{2}, \omega_{1}+2 \omega_{2},\left|2 \omega_{1}-\omega_{2}\right|,\left|2 \omega_{2}-\omega_{1}\right|\right\} .
\end{aligned}
$$

If we consider notation (38), we have the following relation

$$
3\left(\omega_{2} t+\theta_{2}\right)=\omega_{1} t+\theta_{1}-\Delta \psi
$$

By substituting (89) and by taking account of properties (87) and (88), the function (18) can be developed as follows

$$
\begin{aligned}
f= & \left.\varphi_{1}\left\{\frac{\varphi_{3} \omega_{2} a_{2}^{3}}{4 \varphi_{1}} \sin \left(\omega_{1} t+\theta_{1}-\Delta \psi\right)\right)-\omega_{1} a_{1}\left(1-\frac{\varphi_{3}}{\varphi_{1}}\left(\frac{a_{1}^{2}}{4}+\frac{a_{2}^{2}}{2}\right)\right) \sin \left(\omega_{1} t+\theta_{1}\right)\right\} \\
& +\sum_{\omega_{1} \not \omega_{\ell}}^{6}\left(H_{\ell 1} \sin \left(\omega_{\ell} t+\theta_{\ell}\right)+G_{\ell 1} \cos \left(\omega_{\ell} t+\theta_{\ell}\right)\right) \\
= & \varphi_{1}\left\{\frac{\varphi_{3} \omega_{2} a_{2}^{3}}{4 \varphi_{1}}\left[\sin \left(\omega_{1} t+\theta_{1}\right) \cos (\Delta \psi)-\cos \left(\omega_{1} t+\theta_{1}\right) \sin (\Delta \psi)\right]-\omega_{1} a_{1}\left(1-\frac{\varphi_{3}}{\varphi_{1}}\left(\frac{a_{1}^{2}}{4}+\frac{a_{2}^{2}}{2}\right)\right) \sin \left(\omega_{1} t+\theta_{1}\right)\right\} \\
& +\sum_{\omega_{1} \approx \omega_{\ell}}^{6}\left(H_{\ell 1} \sin \left(\omega_{\ell} t+\theta_{\ell}\right)+G_{\ell 1} \cos \left(\omega_{\ell} t+\theta_{\ell}\right)\right)
\end{aligned}
$$


which is in the form (5). Thus for $x_{1}$ we obtain

$$
\left\{\begin{array}{l}
H_{11}=-\varphi_{1}\left[\omega_{1} a_{1}\left(1-\frac{\varphi_{\nu 3}}{\varphi_{v 1}}\left(\frac{a_{1}^{2}}{4}+\frac{a_{2}^{2}}{2}\right)\right)-\frac{\varphi_{3} \omega_{2} a_{2}^{3}}{4 \varphi_{1}} \cos (\Delta \psi)\right] \\
G_{11}=-\frac{\varphi_{3} \omega_{2} a_{2}^{3}}{4} \sin (\Delta \psi) .
\end{array}\right.
$$

Let's now compute the K-B approximation of $x_{2}$. In the case where $\omega_{1}$ is close to $3 \omega_{2}$, we have the following proprieties

$$
\begin{aligned}
& \omega_{2} \approx\left\{\left|2 \omega_{2}-\omega_{1}\right|\right\}, \\
& \omega_{2} \not \approx\left\{\omega_{1}, 3 \omega_{1}, 3 \omega_{2}, 2 \omega_{1}+\omega_{2}, \omega_{1}+2 \omega_{2},\left|2 \omega_{1}-\omega_{2}\right|\right\} .
\end{aligned}
$$

If we consider the notation (38), we have the following relation

$$
\left(2 \omega_{2}-\omega_{1}\right) t+2 \theta_{2}-\theta_{1}=-\left(\omega_{2} t+\theta_{2}\right)-\Delta \psi
$$

By replacing (94) in (18) and taking into account (92) and (93), one obtains

$$
\begin{aligned}
f= & -\varphi_{1}\left\{\omega_{2} a_{2}\left(1-\frac{\varphi_{3}}{\varphi_{1}}\left(\frac{a_{2}^{2}}{4}+\frac{a_{1}^{2}}{2}\right)\right) \sin \left(\omega_{2} t+\theta_{2}\right)+\frac{\left(2 \omega_{2}-\omega_{1}\right) \varphi_{3} a_{2}^{2} a_{1}}{4 \varphi_{1}} \sin \left(\omega_{2} t+\theta_{2}+\Delta \psi\right)\right\} \\
& +\sum_{\omega_{2} \not \omega_{\ell}}^{6}\left(H_{\ell 2} \sin \left(\omega_{\ell} t+\theta_{\ell}\right)+G_{\ell 2} \cos \left(\omega_{\ell} t+\theta_{\ell}\right)\right) \\
= & -\varphi_{1}\left[\omega_{2} a_{2}\left(1-\frac{\varphi_{3}}{\varphi_{1}}\left(\frac{a_{2}^{2}}{4}+\frac{a_{1}^{2}}{2}\right)\right)+\frac{\left(2 \omega_{2}-\omega_{1}\right) \varphi_{3} a_{2}^{2} a_{1}}{4 \varphi_{1}} \cos (\Delta \psi)\right] \sin \left(\omega_{2} t+\theta_{2}\right) \\
& +\frac{\left(\omega_{1}-2 \omega_{2}\right) \varphi_{3} a_{2}^{2} a_{1}}{4} \sin (\Delta \psi) \cos \left(\omega_{2} t+\theta_{2}\right)+\sum_{\omega_{2} \not \omega_{\ell}}^{6}\left(H_{\ell 2} \sin \left(\omega_{\ell} t+\theta_{\ell}\right)+G_{\ell 2} \cos \left(\omega_{\ell} t+\theta_{\ell}\right)\right)
\end{aligned}
$$

Therefore, one deduces

$$
\left\{\begin{array}{l}
H_{22}=-\varphi_{1}\left[\omega_{2} a_{2}\left(1-\frac{\varphi_{3}}{\varphi_{1}}\left(\frac{a_{2}^{2}}{4}+\frac{a_{1}^{2}}{2}\right)\right)+\frac{\left(2 \omega_{2}-\omega_{1}\right) \varphi_{3} a_{2}^{2} a_{1}}{4 \varphi_{1}} \cos (\Delta \psi)\right] \\
G_{22}=\frac{\left(\omega_{1}-2 \omega_{2}\right) \varphi_{3} a_{2}^{2} a_{1}}{4} \sin (\Delta \psi) .
\end{array}\right.
$$

Assembling (91) and (96) and applying the rules (3), yields the approximations given in lemma 3.

\section{K-B approximation for asynchronous force}

The lemma 4 presents results obtained from K-B approximation. Indeed, replacing

$$
\left\{\begin{array}{l}
z_{i}=a_{i} \cos \left(\omega_{i} t+\theta_{i}\right), \\
\frac{d z_{i}}{d t}=-a_{i} \omega_{i} \sin \left(\omega_{i} t+\theta_{i}\right),
\end{array}(i=1,2)\right.
$$

in (47), on obtains after simplifications

$$
\begin{aligned}
f= & -\varphi_{1} \omega_{1} a_{1}\left[1-\frac{\varphi_{3}}{2 \varphi_{1}}\left(\frac{B_{v}}{\triangle_{1}}+\frac{B_{v}}{\triangle_{2}}\right)^{2}-\frac{\varphi_{3}}{\varphi_{1}}\left(\frac{a_{1}^{2}}{4}+\frac{a_{2}^{2}}{2}\right)\right] \sin \left(\omega_{1} t+\theta_{1}\right)-\varphi_{1} \omega_{2} a_{2}\left[1-\frac{\varphi_{3}}{2 \varphi_{1}}\left(\frac{B_{v}}{\triangle_{1}}+\frac{B_{v}}{\triangle_{2}}\right)^{2}\right. \\
& \left.-\frac{\varphi_{3}}{\varphi_{1}}\left(\frac{a_{2}^{2}}{4}+\frac{a_{1}^{2}}{2}\right)\right] \sin \left(\omega_{2} t+\theta_{2}\right)+\sum_{\omega_{\ell} \nsucc \omega_{1} \wedge \omega_{2}}^{r}\left(H_{\ell} \sin \left(\omega_{\ell} t+\theta_{\ell}\right)+G_{\ell} \cos \left(\omega_{\ell} t+\theta_{\ell}\right)\right) .
\end{aligned}
$$

From which, one deduces easily the approximation of lemma 4. 


\section{E K-B approximation for synchronous force}

Consider the case where conditions (20) and (50) are respected. Using substitution (45) with $i=1$ (without substitution of $x_{2}$ ), the procedure presented in asynchronous case (of section 5.1) can applied for finding the approximation of $x_{1}$

$$
\begin{aligned}
& x_{1}=a_{1} \cos \left(\omega_{1} t+\theta_{1}\right)+\frac{B_{v}}{\triangle_{1}} \cos \left(\omega_{v} t\right) \\
& \text { with } \\
& \left\{\begin{array}{l}
\frac{d a_{1}}{d t}=\frac{\varphi_{1} a_{1}}{2}\left(1-\frac{\varphi_{3}}{2 \varphi_{1}}\left(\frac{B_{v}}{\triangle_{1}}\right)^{2}-\frac{\varphi_{3}}{\varphi_{1}}\left(\frac{a_{1}^{2}}{4}+\frac{a_{2}^{2}}{2}\right)\right), \\
\frac{d \theta_{1}}{d t}=0 .
\end{array}\right.
\end{aligned}
$$

Other hand for $x_{2}$, the application of K-B approximation need to modify the second equation of (42) in order to find solution of the form (51). The modification consists to set equation governed $x_{2}$ in near conservative system form (1) with frequency $\omega_{v}$. The substraction of $\triangle_{2} x_{2}$ in both hand of second equation of (42) yields

$$
\frac{d^{2} x_{2}}{d t^{2}}+\omega_{v}^{2} x_{2}=\frac{d}{d t}\left(\varphi_{0}+\varphi_{1}\left(x_{1}+x_{2}\right)-\frac{\varphi_{3}}{3}\left(x_{1}+x_{2}\right)^{3}\right)-\triangle_{2} x_{2}+B_{v} \cos \left(\omega_{v} t\right),
$$

which correspond to perturbation term

$$
f_{2}=\frac{d}{d t}\left(\varphi_{1}-\varphi_{3}\left(x_{1}+x_{2}\right)^{2}\right)\left(\frac{d x_{1}}{d t}+\frac{d x_{2}}{d t}\right)-\triangle_{2} x_{2}+B_{v} \cos \left(\omega_{v} t\right) .
$$

By replacing

$$
\begin{cases}x_{1}=a_{1} \cos \left(\omega_{1} t+\theta_{1}\right), & \frac{d x_{1}}{d t}=-\omega_{1} a_{1} \sin \left(\omega_{1} t+\theta_{1}\right) \\ x_{2}=a_{2} \cos \left(\omega_{v} t+\theta_{2}\right), & \frac{d x_{2}}{d t}=-\omega_{v} a_{2} \sin \left(\omega_{v} t+\theta_{2}\right)\end{cases}
$$

in (100), one obtains

$$
\begin{aligned}
f_{2}= & -\varphi_{1} \omega_{v} a_{2}\left[1-\frac{\varphi_{3}}{\varphi_{1}}\left(\frac{a_{2}^{2}}{4}+\frac{a_{1}^{2}}{2}\right)\right] \sin \left(\omega_{v} t+\theta_{2}\right)-\triangle_{2} a_{2} \cos \left(\omega_{v} t+\theta_{2}\right)+B_{v} \cos \left(\omega_{v} t+\theta_{2}-\theta_{2}\right) \\
& +\sum_{\omega_{\ell} \neq \omega_{2}}^{r}\left(H_{\ell} \sin \left(\omega_{\ell} t+\theta_{\ell}\right)+G_{\ell} \cos \left(\omega_{\ell} t+\theta_{\ell}\right)\right) \\
= & \left\{B_{v} \sin \left(\theta_{2}\right)-\varphi_{1} \omega_{v} a_{2}\left[1-\frac{\varphi_{3}}{\varphi_{1}}\left(\frac{a_{2}^{2}}{4}+\frac{a_{1}^{2}}{2}\right)\right]\right\} \sin \left(\omega_{v} t+\theta_{2}\right)+\left\{B_{v} \cos \left(\theta_{2}\right)-\triangle_{2} a_{2}\right\} \cos \left(\omega_{v} t+\theta_{2}\right) \\
& +\sum_{\omega_{\ell} \neq \omega_{v}}^{r}\left(H_{\ell} \sin \left(\omega_{\ell} t+\theta_{\ell}\right)+G_{\ell} \cos \left(\omega_{\ell} t+\theta_{\ell}\right)\right)
\end{aligned}
$$

Using this expression and rule (3), one gets the approximation

$$
\begin{aligned}
& x_{2}=a_{2} \cos \left(\omega_{v} t+\theta_{2}\right) \\
& \text { with } \\
& \left\{\begin{array}{l}
\frac{d a_{2}}{d t}=\frac{\varphi_{1} a_{2}}{2}\left(1-\frac{\varphi_{3}}{\varphi_{1}}\left(\frac{a_{2}^{2}}{4}+\frac{a_{1}^{2}}{2}\right)\right)-\frac{B_{v}}{2 \omega_{v}} \sin \left(\theta_{2}\right), \\
\frac{d \theta_{2}}{d t}=-\frac{B_{v}}{2 \omega_{v} a_{2}} \cos \left(\theta_{2}\right)+\frac{\triangle_{2}}{2 \omega_{v}} .
\end{array}\right.
\end{aligned}
$$

Assembling (98) and (103), one finds the approximations enounced in lemma 5. 


\section{References}

[1] A.M. Annaswamy and A.F. Ghoniem. Active control of combustion instability: Theory and practice. IEEE Control Systems Magazine, 22(6):37-54, Dec 2002.

[2] L.L. Beranek and I.L. Ver. Noise and Vibration Control Engineering: Principles and Applications. Wiley, New York, 1992.

[3] N.N. Bogoliubov and Y.A. Mitropolski. Asymptotic Methods in the Theory of Nonlinear Oscillations. Hindustan Publishing Corp, Delhi, and Gordon and Breach, New York, 1961.

[4] F. Bouziani, I. D. Landau, and R. R. Bitmead. Quenching oscillations in combustion instabilities using modelbased closed-loop multiplicative control. European Control Conference, Kos, 2007.

[5] F. Bouziani, I. D. Landau, R. R. Bitmead, and A. Voda-Besançon. Analysis of a tractable model for combustion instability: the effect of delay and low pass filtering. Conference on Decision and Control, San Diego, (45), 2006.

[6] E. Camacho, R. Rand, and H. Howland. Dynamics of two van der Pol oscillators coupled via a bath. International Journal of Solids and Structures, 41:2133-2143, 2004.

[7] W. J. Dunstan. System Identification of Nonlinear Resonant Systems. PhD thesis, University of California, San Diego, 2003.

[8] K. Grudziński and J. J. Żebrowski. Modeling cardiac pacemakers with relaxation oscillators. Physica A, 336:153-162, 2004.

[9] C. Hayashi. Nonlinear Oscillations in Physical Systems. McGraw-Hill Book Co, New York, 1964. (reprinted by Princeton University Press, 1985).

[10] H.G. Enjieu Kadji, J.B. Chabi Orou, and P. Woafo. Synchronization dynamics in a ring of four mutually coupled biological systems. Communications in Nonlinear Science and Numerical Simulation (2007), doi:10.1016 / j.cnsns.2006.11.004.

[11] P. S. Landa. Nonlinear Oscillations and Waves in Dynamical Systems. Kluwer, 1996.

[12] P. S. Landa. Regular and Chaotic Oscillations. Springer, New York, 2000.

[13] I. D. Landau and R. R. Bitmead. On the method of Krylov and Bogoliubov for the analysis of nonlinear oscillations. Technical report, Mechanical and Aerospace Engineering Department, University of California, San Diego, 9500 Gilman Drive, La Jolla CA 92093-0411, USA, Jan 2004.

[14] Ü. Lepik and H. Hein. On response of nonlinear oscillators with random frequency of excitation. Journal of Sound and Vibration, 288:275-292, 2005. 
[15] H.K. Leung. Synchronization dynamics of coupled van der Pol systems. Physica A, 321:248255, 2003.

[16] K. Rompala, R. Rand, and H. Howland. Dynamics of three coupled van der Pol oscillators with application to circadian rhythms. Communications in Nonlinear Science and Numerical Simulation, 12:794-803, 2007.

[17] B. Shapiro and B.T. Zinn. High frequency and nonlinear vibrational control. IEEE Transactions on Automatic Control, Jan 1997.

[18] J.-J. Sinou and L. Jézéquel. The influence of damping on the limit cycles for a self-exciting mechanism. Journal of Sound and Vibration, 304:875-893, 2007. 